\title{
Prioritization of abiotic and biotic stress responses by direct linkage of ABI1 phosphatase and CPK5 calcium-dependent protein kinase
}

ABI1 - CPK5 switch prioritizes plant stress responses

Heike Seybold ${ }^{\mathrm{a}, 1}$, Jennifer Bortlik ${ }^{\mathrm{a}, 2}$, Benjamin Conrads ${ }^{\mathrm{a}}$, Wolfgang Hoehenwarter ${ }^{\mathrm{b}}$, Tina Romeis $^{\mathrm{a}, \mathrm{b}, 3}$

a Department of Plant Biochemistry, Dahlem Centre of Plant Sciences, Institute for Biology, Freie Universität Berlin, 14195 Berlin, Germany

${ }^{\mathrm{b}}$ Leibniz Institute of Plant Biochemistry, Dept. Biochemistry of Plant Interactions, 06120 Halle (Saale), Germany

${ }^{1}$ present address: Christian-Albrechts Universität Kiel, Botanical Institute, Dept. Environmental Genomics, 24118 Kiel, Germany

${ }^{2}$ present address: Leibniz Institute of Vegetable and Ornamental Crops, 14979 Großbeeren, Germany

${ }^{3}$ To whom correspondence should be addressed Email: tina.romeis@ipb-halle.de

\begin{abstract}
In nature plants are constantly challenged by simultaneous abiotic and biotic stresses, and under conflicting stress scenarios prioritization of stress responses is required for plant survival. Calcium-dependent protein kinase CPK5 is a central hub in local and distal immune signaling, required upstream of hormone salicylic acid (SA)-dependent systemic acquired resistance (SAR). Here we show that CPK5 signaling-dependent immune responses are effectively blocked and pathogen resistance is reverted either upon treatment of plants with abscisic acid (ABA) or in genetic mutant backgrounds lacking PP2C phosphatase activities including abil2. Consistently, enhanced immune responses occur upon co-expression of CPK5 kinase with active variants of $\mathrm{ABI} 1$ phosphatase $\mathrm{ABI} 1_{\mathrm{G} 180 \mathrm{~S}}$ and $\mathrm{ABI} 1_{\mathrm{G} 181 \mathrm{~A}}$. Biochemical studies and mass spectrometry-based phosphosite analysis reveal a direct ABI1 phosphatase-catalyzed dephosphorylation of CPK5 at T98, a CPK5 auto-phosphorylation site. CPK5 $98 \mathrm{~A}$, mimicking continuous de-phosphorylation through ABI1, correlates with an increase in kinase activity and CPK5 function in ROS production. CPK5 $5_{\text {T98D }}$, mimicking a CPK5 auto-phosphorylated status
\end{abstract}


under ABA-induced phosphatase inhibition, leads to inactivated CPK5 causative to an immediate stop of immune responses.

Our work reveals an elegant mechanism for plant stress prioritization, where the ABAdependent phosphatase $\mathrm{ABI}$, negative regulator of abiotic responses, functions as positive regulator of biotic stress responses, stabilizing CPK5-dependent immune responses in the absence of ABA. This mechanism allows continuous immune signaling during pathogen survey in environmentally non-challenging conditions. Under severe abiotic stress, immune signaling is discontinued via a direct biochemical intersection through a phosphatase/kinase pair recruiting two key regulatory enzymes of these antagonistic signaling pathways.

\section{Significance Statement}

Plants challenged by simultaneous abiotic and biotic stresses prioritize in conflicting scenarios to guarantee survival. Systemic acquired resistance (SAR), a plant's immune memory, depends on the hormone salicylic acid (SA) and prepares a plant for future pathogen attack. Adaptation to abiotic stress signaling involves the hormone abscisic acid (ABA). We identify a direct biochemical mechanism by which ABA-mediated abiotic signaling prioritizes over immune responses via reversible phosphorylation involving two key regulatory enzymes of these antagonistic pathways. Phosphatase ABI1, negative regulator of abiotic stress signaling, dephosphorylates calcium-dependent protein kinase CPK5 at an auto-phosphorylation protein mark, leading to kinase activation and function in immune signaling. Under abiotic stress ABAdependent phosphatase inhibition results in continuous phosphorylated inactive kinase, preventing immune signaling.

\section{Introduction}

Plants experience a continuously changing environment and must permanently adapt to new conditions. These encompass abiotic changes like temperature variations, shortage in water supply, or high salinity, and biotic challenges, where plants have to tackle attacks by various microbial pathogens or herbivorous insects. Because stress responses are energy consuming and often come to the cost of a retardation or a full stop of growth, it is in the interest of a plant to implement mechanisms to limit specific stress responses in time and to prioritize between multiple simultaneous external challenges.

Pathogen infections lead to a rapid activation of the plant immune system. Plants perceive attacking microbes in a non-species-specific manner via molecules, Pathogen-Associated 
Molecular Patterns (PAMPs), which bind as ligands to corresponding receptors (PatternRecognition Receptors, PRRs) on the cell surface, intracellular defense reactions become initiated and lead to PAMP-triggered immunity (PTI). These cytoplasmic reactions include an increase in the concentration of calcium ions $\left(\mathrm{Ca}^{2+}\right)$, the activation of protein kinases including $\mathrm{Ca}^{2+}$-decoding calcium-dependent protein kinases (CDPKs), production of reactive oxygen species (ROS), phytohormone signaling, transcriptional reprogramming, and changes in metabolites to ultimately accomplish pathogen resistance (1). A second layer of defense has evolved in plants, Effector-Triggered Immunity (ETI), which detects PTI-suppressing effectors from adapted pathogens. Intracellular recognition inducing ETI results in the induction of a similar set of intracellular reactions compared to PTI, except immune responses are stronger and long-lasting and lead to a hypersensitive cell death. Furthermore, PTI and ETI may prime a systemic long-term immune memory throughout the plant. During the state of systemic acquired resistance (SAR) a phytohormone salicylic acid (SA)-dependent immune status is maintained, which renders the plants prepared for a consecutive second pathogen attack (2-4). Calcium-dependent protein kinase CPK5 from Arabidopsis is a highly $\mathrm{Ca}^{2+}$-sensitive CDPK expressed in plant mesophyll cells and functions as a key regulatory enzyme in plant immunity (5-8). CPK5 contributes to local pathogen resistance, to defense signal spread, and to the onset and maintenance of long-term systemic resistance, where CPK5 functions upstream of SA- and NHP-dependent SAR. Plants of enhanced CPK5-signaling are more resistant to virulent Pseudomonas syringae pv. tomato (Pto) DC3000 in PTI and show an 'enhanced SAR' phenotype in ETI (9). These plants accumulate high levels of SA and are characterised by stunted growth with developing lesions, which are typical characteristics for a constitutive activated immune system $(6,9)$. Consistently, plants that carry mutations in the CPK5 gene coding for non-functional protein variants in different $c p k 5$ alleles do not show prolonged immunity in an NLR-dependent autoimmune phenotype, and a reversion to wild-type growth is observed (10). Thus, in the biological context, a plant may stop immune signaling, turn off its immune system, and release SAR by a mechanism, which involves the direct biochemical inactivation of the CPK5 enzyme. CDPKs are modular enzymes, in which a protein kinase effector domain is via a pseudo-substrate region is directly linked to a calcium-binding sensor domain with, as in CPK5, four consensus EF-hand $\mathrm{Ca}^{2+}$-binding motifs (11-15). Direct binding of $\mathrm{Ca}^{2+}$ - has been correlated with a conformational change which is a prerequisite for enzyme activity and its subsequent catalysis of auto- and substrate phosphorylation (6, 16-21). Remarkably, the activation threshold for CPK5 has been determined to $\mathrm{Kd} 50\left[\mathrm{Ca}^{2+}\right]$ of $100 \mathrm{nM}$, an intracellular $\mathrm{Ca}^{2+}$ concentration close to a plant cell's resting state (9). This low $\mathrm{Ca}^{2+}$ value 
implies that a gradual withdrawal of $\mathrm{Ca}^{2+}$ may be insufficient for a rapid biochemical inactivation of the catalytic CPK5 activity and demands for additional molecular modifications.

Abscisic acid (ABA) is the key regulatory phytohormone in the control of abiotic stress tolerance, causal to the induction of rapid stomatal closure and influencing developmental growth processes $(22,23)$. The control of ABA function depends on the biosynthesis of the phytohormone, on the presence of ABA receptor proteins of the RCAR/PYL protein, and on downstream signaling components. Key regulators of the ABA signal transduction pathways involve clade 2 phosphatases (PP2Cs) such as ABI1 or its close homologues ABI2, HAB1 and PP2CA, and protein kinases such as OST1 and other $\left(\mathrm{Ca}^{2+}\right.$-regulated) protein kinases $(24,25)$. In the absence of an abiotic challenge to the plant, phosphatase ABI1 functions as a negative regulator of ABA signaling by de-phosphorylating and thereby inactivating OST1. Drought and increased $\mathrm{ABA}$ levels lead to an ABA/RCAR-mediated sequestration and inactivation of the PP2C phosphatase, to a subsequent release of phosphorylated active OST1 kinase, and to a further progression of ABA signal transduction (26-28). In guard cells, membrane located ion channels are employed as immediate early recipients of ABA signaling, which mediate ion fluxes changes-dependent stomatal closure (29-31).

When exposed simultaneously to biotic pathogen and abiotic water supply stress, drought may represent a considerably higher threat to the whole plant compared to a pathogen infection that is usually limited to a part of the plant. Accordingly, after invasion of the pathogen into the plant tissue, $\mathrm{ABA}$ is suppressing plant defense responses. Exogenous application of $\mathrm{ABA}$ increased the susceptibility of plants to bacterial as well as fungal pathogens (32-38). Consistently, ABA-deficient or -insensitive mutants were more resistant towards (hemi-) biotrophic pathogens $(35-37,39)$. It seems logical, that components of ABA biosynthesis and -signaling pathway were identified among plant genes that were induced by secreted type III effectors upon bacterial infection with strain Pto DC3000, aiming for a pathogen advantage (37). Likewise, a gene coding for the rate-limiting step in ABA biosynthesis was induced by infection of wheat with wheat pathogen Xanthomonas translucens (40).

Interestingly, a different picture emerges considering the ABA-dependent closure of guard cells, which is an important layer of PAMP-induced immune responses against invading Pto DC3000 on the pre-invasive level (41-44). In response to PAMPs stomata close within one hour. The pathogen virulence factor coronatine triggers a re-opening, facilitating pathogen re- 
entry into the host plant (45), whereas overexpression of the ABA-receptor RCAR3 prohibits the coronatine-mediated stomatal re-opening and leads to increased resistance against Pto DC3000 upon surface inoculation (46). Consistently, pp2ca mutant plants, lacking a negative regulatory phosphatase and in such mimicking constitutive ABA signaling, are more resistant to Pto DC3000 when inoculated by dipping (46). Furthermore, in guard cells parallel integration of SA- and ABA-signaling upstream of stomatal closure were observed $(47,48)$. Although such phytohormone-directed control of stomatal closure may contribute to pre-invasive immunity their mutual participation in a guard cell-based long-lasting resistance seems unlikely because prolonged stomatal closure prohibits gas exchange, and key functions in guard cell physiology with respect to photosynthesis and transpiration will be compromised (31). Mesophyll cellbased post-invasive immunity leads to more long-term and systemic pathogen resistance.

Trade-offs between biotic and abiotic stress responses allow a plant to prioritize the abiotic over the biotic stress responses (49). Mechanisms for prioritization against immune response may target rather early stages at the level of perception, for example through a control of the secretion and accessibility of PRR receptors in the PM (50), or at the level of signaling, for example through ABA-induced transcriptional activation of phosphatase genes, whose gene products catalyze an inactivation of immune-associated MAP kinases (51). Stress prioritization may also involve rather late stages, for example employing the phytohormone-mediated crosstalk, where ABA signaling suppresses SA synthesis and signaling and in such prohibiting SAdependent immunity $(52,53)$.

Here we decipher a novel direct biochemical mechanism, which mediates the switch from biotic to abiotic stress responses. ABI1-catalyzed de-phosphorylation of an auto-phosphorylation site of CPK5 links the two antagonistic stress response pathways at respective signaling hubs CPK5, a master regulator promoting biotic systemic SA-dependent immune signaling, and the phosphatase $\mathrm{ABI}$, a negative regulator of abiotic ABA-signaling, and in the absence of abiotic challenges positive regulator of immune signaling.

\section{Results}

\section{ABA reverts CPK5 signaling-dependent immune phenotype and inhibits CPK5 activity}

Enhanced CPK5 signaling in CPK5-YFP \#7 overexpressing plants leads to an increase in bacterial resistance in young plants as well as to SA-dependent long-term immunity and enhanced SAR $(3,6,9,10)$. Furthermore, in later plant stages enhanced CPK5 signaling correlates with a reduced plant rosette size and a SA-dependent lesion mimic phenotype (Fig. 
S1A-C). Significant size differences became evident from day 33 and further increased with plant age (Fig. S1A). Interestingly, continuous treatment with $10 \mu \mathrm{M}$ ABA over several weeks with ABA application every second day reverted all CPK5 signaling-dependent growth phenotypes of CPK5-YFP \#7, and plants were of wild type size with no or only few lesions (Fig. 1A-D, Fig. S1C). Also, transcript levels of the SA biosynthesis and signaling marker genes ICS1 and PR1, and of SA-independent CPK5 marker gene NHL1O were completely reverted (Fig. 1 E-G). Basal endogenous ABA phytohormone levels remained unaltered in CPK5-YFP \#7 plants indicating that these endogenous ABA levels are independent of elevated SA levels (Fig. S2).

We next assessed whether ABA treatment directly effects CPK5 enzyme activity. Protein extracts originating from ABA-treated CPK5-YFP \#7 plants showed strongly reduced CPK5 phosphorylation activity in in-gel kinase assays. The differences in enzyme activity became detectable as early as two hours after treatment in a short time course (Fig. 1H), and accumulated over time to up to 14 days investigated compared to untreated plants (Fig. 1I), while CPK5-YFP protein levels in ABA-sprayed plants stayed comparable to mock treatment.

\section{$\mathrm{ABI1}$ regulates CPK5 function and activity}

To assess mechanistically if ABA signaling-associated PP2C-type of protein phosphatases are involved the inactivation of CPK5, we generated crosses between CPK5-YFP \#7 and mutant lines abil-2, abi2-2, hab1-1, and pp2ca, lacking respective protein phosphatase activities of $\mathrm{ABI} 1, \mathrm{ABI}$, HAB1, and PP2Ca. CPK5-YFP protein expression in the resulting lines has been confirmed by western blot and by detection of the eYFP transcripts (Fig. S3B,C). In the absence of these phosphatase activities, elevated transcript levels of PRI and NHL1O and the lesion mimic phenotype, both hallmark for enhanced CPK5 immune signaling, was reverted in all lines (Fig. 2A,B and Fig. S3A,D). In bacterial growth assays, the CPK5-dependent constitutive resistance to bacterial pathogens Pto DC3000 in CPK5-YFP \#7 was entirely reverted or at least partially lost to wild type when CPK5-YFP is expressed in the genetic backgrounds of abil-2 (Fig. 2D and Fig. S3E), abi2-2 (Fig. S3E) or habl-1 and pp2ca-1 (Fig. 2D). Consistently, a reduction of rapid flg22-induced ROS generation occurs in the phosphatase mutant background reminiscent to $c p k 5$ mutant plants (Fig. 2D) (6). These data suggest that phosphatase activity is beneficial to CPK5 function.

To address this positive effect directly, we co-expressed active ABI1 phosphatase variants and CPK5 kinase together with NADPH oxidase RBOHD transiently in N. benthamiana and 
compared the levels of CPK5-dependent RBOHD-catalyzed ROS production in the absence of the phosphatase (6). We used the truncated constitutive active variant CPK5-VK (6), lacking its regulatory $\mathrm{CAD}$, which is a biochemically active enzyme independent of a further stimulation. Constitutive phosphatase activity mimicking the absence of ABA was achieved using two gain-of-function ABA insensitive phosphatase variants derived from the semidominant abil-1 and abi1-11 alleles. ABI1-1 carries the amino acid substitution G180D (54, 55) and ABI1-11 the substitution G181S (56).The presence of both phosphatase variants, ABI11 and ABI1-11, together with CPK5-VK induced a dramatic increase in ROS production (Fig. $3 \mathrm{~A})$, which also is evident in the absence of ectopic RBOHD, relying on endogenous RBOH enzymes. No ROS production occurs when these phosphatase variants ABI1-1 and ABI1-11 are combined with RBOHD alone, indicating that the positive ABI phosphatase effect requires CPK5. We observed consistent results in Arabidopsis mesophyll protoplasts upon coexpression of ABI1-1 or ABI1-11 together with CPK5 full-length enzyme, and we assessed CPK5 kinase activity. Both, in the absence and in the presence of flg22 stimulation, a stabilizing and enhancing effect of the ABI1 phosphatases on CPK5 activity occurred (Fig. 3B).

\section{ABI1 positively influences CPK5-dependent immunity}

To further study the ABI1/CPK5 relation we generated stable transgenic lines which co-express phosphatase mCherry fusion proteins of ABI1-1-mCh and ABI1-11-mCh together with CPK5YFP by dipping the ABI1 constructs into the CPK5-YFP \#7 background. As appropriate controls, we transformed Col-0 and cpk5 mutant with these ABI1 constructs. Co-expression of ABI1-1 with CPK5-YFP resulted in severely dwarfed seedlings, which rarely survived. Plant lines co-expressing ABI1-11-mCh together with CPK5-YFP were viable. The expression of proteins ABI1-11-mCh and CPK5-YFP was verified by western blot in all lines (Fig. S4A) and by quantitative Real-time PCR (qPCR) (Fig. S4B). Co-expression of ABI1-11-mCh with CPK5-YFP resulted in a more severe lesion mimic phenotype (Fig. S4C) accompanied by strongly increased PRI and NHL1O transcript levels (Fig. 3C), even further enhanced in dwarfed ABI1-1-mCh $x$ CPK5-YFP plants (Fig. S5A). In bacterial growth assays both CPK5YFP \#7 and ABI1-11-mCh $x$ CPK5-YFP were equally resistant to Pto DC3000 (Fig. 3D). In contrast, plants expressing ABI1-11-mcherry in cpk5 or Col-0 background showed wildtypelike susceptibility.

\section{ABI1 increases CPK5 activity by direct de-phosphorylation at auto-phosphorylation site T98}


We next assessed whether CPK5 is a functional direct substrate for ABI1 for dephosphorylation. Recombinant tagged CPK5 protein was subjected to autocatalysis. Kinase activity was then inhibited by K252-a, and the reaction was divided and subsequently further incubated over a time course in the presence of either active or heat-inactivated ABI1 phosphatase. Active phosphatase reduced the CPK5 auto-phosphorylation signal up to $40 \%$ within 60 minutes (Fig. 4A). No comparable decrease in band intensity occurred with heatinactivated ABI1. To identify amino acids targeted by ABI1-catalyzed de-phosphorylation in CPK5 we repeated the experiment with CPK5-YFP followed by mass spectrometry-based differential phosphosite analysis. Because ABI1 triggered an increase in ROS production together with the CPK5-VK variant (Fig. 3A) we focused on the N-terminal part of the CPK5 protein encompassing the variable and the kinase domain. From the four detected phosphorylated amino acids at S8, T98, S100 and S338 only T98 revealed a differential phosphorylation pattern with a decrease in intensity in the presence of active phosphatase (Fig. 4B, Fig. S6). We therefore selected T98 as the crucial amino acid that may mediate a phosphorylation status-dependent control of CPK5 enzyme activity.

To verify the importance of T98 we generated CPK5 variants carrying the amino acid substitutions T98A (mimicking constant de-phosphorylation through ABI1), and T98D (mimicking constant CPK5 auto-phosphorylation in the absence of (active) ABI1) and enzyme activity was assessed in a transient ROS assay in $N$. benthamiana as shown in Fig. 3A. Remarkably, a dramatic increase in transient ROS production is observed with CPK5 T98A alone, in the absence of the phosphatase, to the level of CPK5-VK (wild-type sequence) and ABI1-1 co-expression, and no further increase was obtained in the presence of ABI1-1 (Fig. 4C). In contrast, no ROS production occurs with the CPK5 T98D variant, also not in the presence of the ABI1-1 phosphatase. These data confirm the identification of T98 as a crucial amino acid in CPK5, which directs CPK5 activity and function in immune signaling, and in such may present a mechanistic switch between biotic vs. abiotic stress responses.

\section{Discussion}

Plant survival depends on stress response activation and inactivation, which under simultaneous multiple stress challenges facilitates prioritization between different abiotic and biotic danger scenarios. Here we have identified a pair of key regulatory proteins, the calcium sensor protein kinase CPK5, positive regulator of immune signaling, and protein phosphatase ABI1, negative 
regulator of $\mathrm{ABA}$ - and abiotic stress signaling, which are mechanistically linked to orchestrate stress signaling and resource allocation.

CPK5 is a mesophyll cell-expressed enzyme highly responsive to $\mathrm{Ca}^{2+}$ and becomes biochemically activated within $\mathrm{sec}-$ min upon pathogen perception. CPK5 contributes to local defense signal initiation, signal propagation, and to the onset and maintenance of systemic resistance in the entire plant, where CPK5 functions upstream of SA-dependent SAR $(5-7,9)$. Thus, CPK5 represents an ideal surveyor of danger by pathogen attack. Activated CPK5signaling mirrors constitutive defense priming, and consistent with this 'enhanced SAR' phenotype plants display increased resistance to bacterial pathogens. These plants accumulate high levels of SA causative for stunted growth and lesion development $(6,9)$.

Here we show that CPK5-dependent immunity requires phosphatase activity. In the absence of PP2C phosphatase activities in respective phosphatase mutant abil-2, abi2-2, habl-1, and pp2ca-1 backgrounds CPK5 signaling-dependent immune responses are entirely lost. These responses include CPK5-mediated ROS production, NHL10 marker gene expression as well as plant growth retardation and the lesion mimic phenotype (Fig. 2A,B). In accordance, CPK5dependent enhanced resistance is either completely lost (lines CPK5-YFP x abil-2, CPK5-YFP $x$ abi2-2) or is reduced (CPK5-YFP $x$ habl-1 and CPK5-YFP $x$ pp2ca-1) (Fig. 2D and Fig. $\mathrm{S} 3 \mathrm{E})$. This data indicates that $\mathrm{ABI} 1$ and $\mathrm{ABI} 2$ are the predominant $\mathrm{PP} 2 \mathrm{C}$ phosphatases in the CPK5-mediated control of onset and maintenance of plant immunity and resistance.

Consistently, we have generated gain-of-function protein variants ABI1-1 and ABI1-11, which carry the G/D and G/A amino acid substitutions analogous to respective described (semi-) dominant alleles (54-56). When co-expressed together with constitutive active CPK5-VK in $N$. benthamiana both variants lead to a dramatic increase of synergistic ROS production (Fig. 3A). In stable transgenic A. thaliana plants expressing CPK5-YFP the co-expression of ABI1-1mcherry results in a stronger effect than ABI1-11-mcherry (Fig. S5A) and leads to tiny plants and lethality for the ABI1-1 variant, reminiscent to a constitutive 'enhanced SAR' phenotype (9) and auto-immunity (10). This finding suggests an over-induced immune response caused by already activated CPK5-signaling plus an additional synergistic factor through phosphatase ABI1-1. ABI1-11-mcherry co-expression yielded viable plants, in which the lesion mimic phenotype of line CPK5-YFP \#7 is further increased. ABI1-11 induces no lesion mimic phenotype in the absence of enhanced CPK5-signaling (Fig. S4C). In accordance with the visible phenotype, CPK5-YFP-dependent PRI and NHL10 transcript levels were increased in 
the presence of ABI1-11-mcherry together with CPK5-YFP but not without the kinase (Fig. 3C). In consequence, ABI1 does not confer resistance without CPK5 (Fig. 3D). Interestingly, pathogen resistance has been correlated with the abil-1 semi-dominant allele compared to its respective Ler wild type (37). In our experiments in Col-0 wild type neither plants which express native ABI1-1 carrying the same amino acid substitution (abil-1 in Col-0) nor overexpressed ABI1-11 (ABI1-11-mCherry in Col-0) alone did show an increased resistance in Pto DC3000 infection assays (Fig. 3D, Fig. S5B).

Because our data implicate a positive regulation of ABI1 phosphatase activity on CPK5 immune signaling and function, we addressed whether ABI1 directly de-phosphorylates CPK5 at T98. Using recombinant enzymes, we could demonstrate that protein phosphatase ABI directly de-phosphorylates CPK5 protein (Fig. 4). (Auto-)phosphorylation of CDPKs, inclusive of CPK5, has often been correlated and interpreted as an activation step, because of its temporal occurrence after stress exposure, for example by flg22 treatment of plants or cells $(6-8,18,19)$. Regulation of CDPKs by phosphorylation in addition to $\mathrm{Ca}^{2+}$ has been considered as a mechanism to fine-tune kinase activation in terms of strength and duration $(14,15,57)$. Interestingly, phospho-proteomic studies had indicated a rapid de-phosphorylation subsequent to ABA treatment of plants at CPK5 S552 close to the protein's C-terminus (58). Here we have identified T98 as a crucial amino acid which is directly susceptible to ABI1-catalyzed dephosphorylation (Fig. 4B), differing from S552, and T98 resides within the kinase domain in CPK5-VK, the enzyme variant sufficient for ABI1-induced ROS production (Fig. 3A, 4C). The functional relevance of T98 was verified by the T98D phosphomimic variant of CPK5-VK, which produced no ROS (Fig 4C), neither in the presence nor in the absence of ABI1. In contrast, T98A cannot be phosphorylated and thereby resembled the fully de-phosphorylated status of CPK5-VK at this site, mimicking ABI1 phosphatase action. Accordingly, high levels of ROS were produced (Fig. 4C).

Thus, our data identify PP2C phosphatase ABI1, which directly catalyzes a de-phosphorylation of calcium-regulated kinase CPK5 protein leading to enzyme activation and promotion of immune signaling. Interestingly, expression of PP2C phosphatase genes HAII, HAI2, and HAI3 is induced in response to $\mathrm{ABA}$, and respective phosphatase proteins can interact with, dephosphorylate and inactivate MAP kinases MPK3 and MPK6, which thereby leads to immune suppression (51). 
PP2C phosphatases, and in particular ABI1, are negatively regulating ABA signaling in the absence of $\mathrm{ABA}$. ABA perception results in inhibition of PP2C activity by the sequestration of the phosphatase proteins through the ABA/RCAR1 complex. In guard cells, ABA signal transduction employs $\mathrm{Ca}^{2+}$-dependent and $\mathrm{Ca}^{2+}$-independent pathways, which merge at S-type anion channel phosphorylation and activation leading to stomatal closure. Interestingly, a SAinduced stomatal closure has been reported, which is compromised in guard cells of $c p k 3$ and cpk6 mutant plants (47), placing SA upstream of CDPK function and independent of ABA. Other data show several distinct calcium-sensor kinases including CPK6, CPK21 or CPK23, enzymes which are integrated into ABA-induced and ion channel-mediated stomata control. Remarkably, these enzymes have a significant higher $\mathrm{Kd} 50\left[\mathrm{Ca}^{2+}\right]$ of $180 \mathrm{nM}(\mathrm{CPK} 6)$, and 1000 $\mathrm{nM}(\mathrm{CPK} 21)$ than what we have observed for CPK5 $\mathrm{Kd} 50\left[\mathrm{Ca}^{2+}\right]$ of $100 \mathrm{nM}$. This low Kd50 $\left[\mathrm{Ca}^{2+}\right]$ may explain why an ectopic expression of CPK5 in protoplasts or a heterologous expression in oocytes lead to similar results to its closest homolog CPK6 in terms of SLAC1activation (59).

Here we show that PP2C phosphatases link ABA- and CPK5-controlled immune signaling. In mesophyll cells, where pathogens spread and where post-invasive immune reactions lead to resistance, the abolition of the negative regulation of $\mathrm{ABA}$ signaling - as in $p p 2 c$ knockout mutant backgrounds lacking phosphatase activities - resembles a constant ABA exposure. Consistently, CPK5-signaling-dependent immune responses were reversed. These include reset of PRI and NHL1O gene expression and reversion of plant growth and lesion mimic phenotype under both, continuous ABA treatment, and in genetic $p p 2 c$ knockout mutant backgrounds (Fig. 1A-D; Fig. 2A; Fig. S1 and Fig. S3A,D). Consequently, enhanced CPK5-dependent pathogen resistance against Pto DC3000 is lost (Fig. 2D and Fig. S3E) despite the presence of CPK5YFP protein (Fig. S3B,C).

Because we show that the endogenous ABA levels are unaltered in CPK5-YFP \#7 compared to wild type (Fig. S2), we can exclude a phytohormone-mediated cross-talk where reduced ABA levels are responsible for high SA-levels and SA-dependent transcript accumulation (6). In contrary, exogenous ABA application not only leads to a reversion of CPK5-signalingmediated defense responses, similar to those observed in the icsl, nprl, fmol or sardl mutant backgrounds (9). Even more, ABA treatment of plants results in rapid and prolonged biochemical in-activation of the CPK5 enzyme itself.

Because CPK5 protein is a central hub for basal and systemic immunity, it represents an ideal direct target for ABA-mediated cross-regulation. CPK5 protein requires initial $\mathrm{Ca}^{2+}$ to allow 
the enzyme to adopt its primary open conformation and to become biochemically active. Due to its low $\mathrm{Kd} 50\left[\mathrm{Ca}^{2+}\right]$ of $100 \mathrm{nM}$, however, it is conceivable that in planta biochemical inactivation of CPK5 depends on additional mechanisms, and a transient withdrawal of $\mathrm{Ca}^{2+}$ may neither be fast nor efficient enough. Thus, CPK5 auto-catalyzed phosphorylation at T98 provides an elegant intrinsic mechanism as a mean to stop CPK5 activity and in thus stop continuation of CPK5-dependent immune signaling. Under environmental non-challenging conditions, in the presence of active PP2C phosphatases such as ABI1, T98 will become constantly de-phosphorylated. This de-phosphorylation allows an immediate switch to resume CPK5 activity and immune signaling, if an endogenous plant survey detects pathogen threat and commands action. Under adverse environmental conditions as for example drought, with phytohormone $\mathrm{ABA}$ in place, the ABA/RCAR1 complex sequesters and inhibits phosphatase ABI1. In such CPK5 stays phosphorylated at T98, remains inactive, and onset and maintaining of immune signaling via CPK5 is prohibited. Interestingly, a specific CPK5 phosphorylation status in the absence of $\mathrm{Ca}^{2+}$ has been proposed in the context of priming (in ETI or in autoimmune responses), which in systemic plant tissue contributes to the immune memory allowing a faster response activation upon a secondary pathogen attack (9). Thus, ABA-dependent dephosphorylation at T98 may be part of a 'de-priming' mechanism of CPK5, enabling the plant to 'forget' previous biotic stimuli. Whether T98 is the only 'protein mark' required for reversible CPK5 phospho-regulation or whether additional sites for example at T544 and S552 are involved remains to be shown.

\section{Conclusion}

Reversible phosphorylation at protein mark T98 of CPK5 provides a biochemical mechanism for a molecular switch between ABA/ABI1-mediated abiotic signaling and CPK5-mediated SA-dependent immune responses. These data not only close a mechanistic gap between these antagonistic pathways. Even more, the reciprocal control of CPK5 kinase activity as a central hub for plant post-invasive immunity allows the plant to prioritize in favor of abiotic stress tolerance despite of an ongoing biotic challenge of pathogen pressure.

\section{Material and Methods}

Detailed descriptions of materials and methods used in this study, including plant growth and treatment conditions, pathogen inoculations, protoplast isolation, protein kinase analyses, gene 
expression analyses, phytohormone analysis and phosphoproteomics, as well as statistics, are provided in SI Appendix, Materials and Methods. All primers used are listed in SI Appendix, Table S1.

\section{Acknowledgements}

We thank Ruth Lintermann for generation of CPK5 amino acid substitution variants and Tobias Lortzing for Phytohormone measurements. Plant lines carrying mutations in $P P 2 C$ phosphatase genes (abi1-1, abil-2, abi2-1, hab1-1, pp2ca-1) were kindly provided by Jörg Kudla (University of Münster) and Erwin Grill (Technical University of Munich). This research was supported by Deutsche Forschungsgemeinschaft (DFG) within Collaborative Research Centre SFB973 to T.R..

\section{Author contributions}

HS and TR designed the research. HS, JB, BC and WH performed the experiments and analyzed the data. HS and TR wrote the manuscript. 

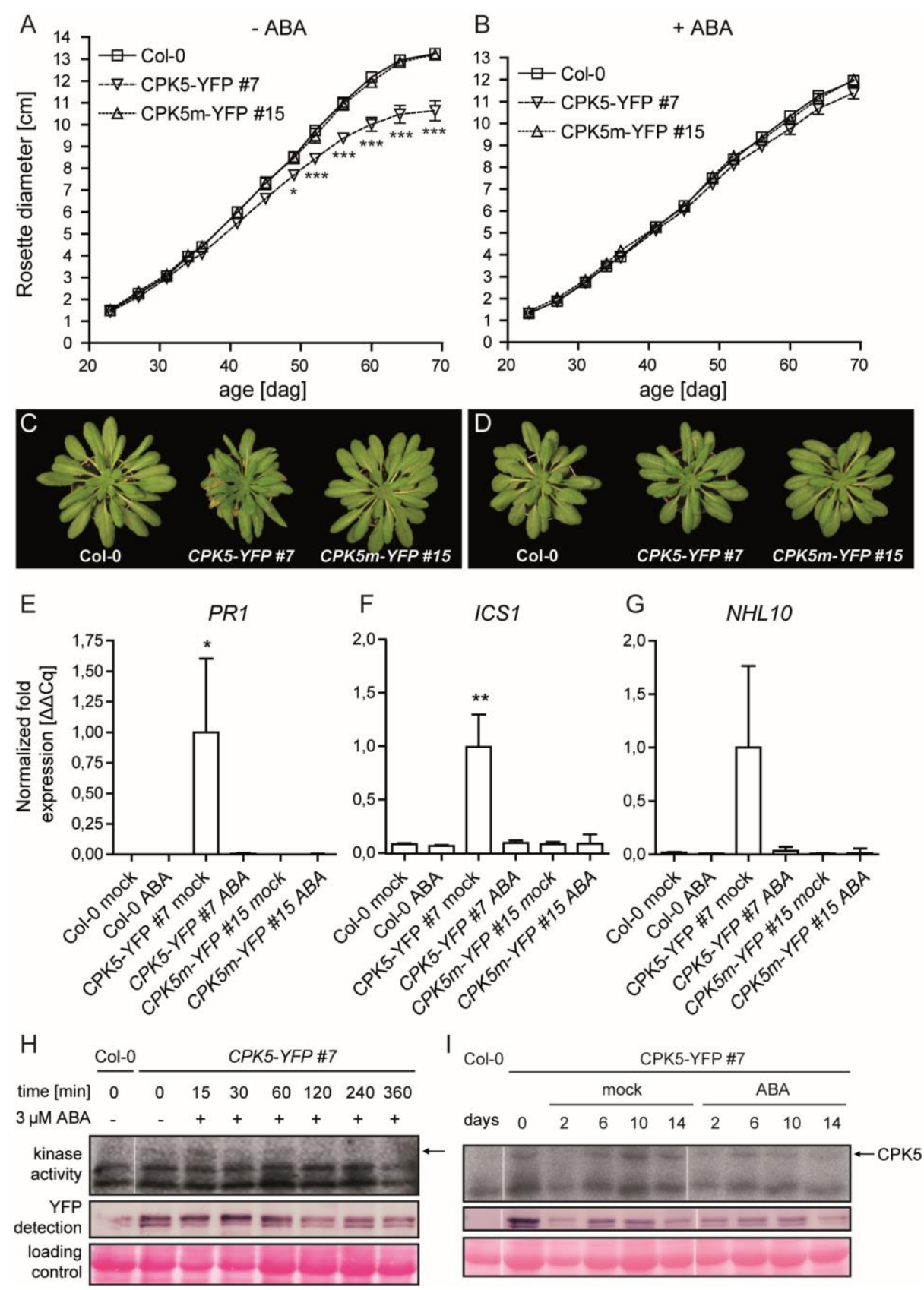

Fig. 1 ABA inhibits the CPK5-YFP phenotype and kinase activity. $(A)-(D)$ Monitoring of rosette diameter during growth phase of Col-0, CPK5-YFP \#7 and CPK5m-YFP \#15 (n = 8-13) over a time course of 10 weeks and corresponding phenotypes of 10 week old plants in the absence $(A)+(C)$ and presence $(B)+(D)$ of treatment with $10 \mu \mathrm{M}$ ABA. $(E)-(G)$ Basal transcript levels of marker genes in 6 week old plants as mentioned in $(A)$ with or without ABA treatment $(\mathrm{n}=4)$. Values represent mean $\pm \mathrm{SD}$ of $\mathrm{n}$ biological replicates. Data in $(A)+(B)$ was checked for statistical differences using two-way ANOVA (Bonferroni post-test). Data in $(E)-(G)$ was checked for statistical differences using one-way ANOVA (Dunnett post-test). Significant differences $*=\mathrm{p}<0.05$, $* *=\mathrm{p}<0.01$ and $* * *=\mathrm{p}<0.001$. $(H)+(I)$ In gel kinase activity in overexpression line CPK5-YFP \#7 after spray treatment with $3 \mu \mathrm{M}$ ABA. Kinase activity was tested $(H)$ over a short a time course of $6 \mathrm{~h}$ after ABA treatment or $(I)$ over a period of 14 days with repeated ABA treatment every second day. Expression of CPK5 was detected via western blot analysis. 

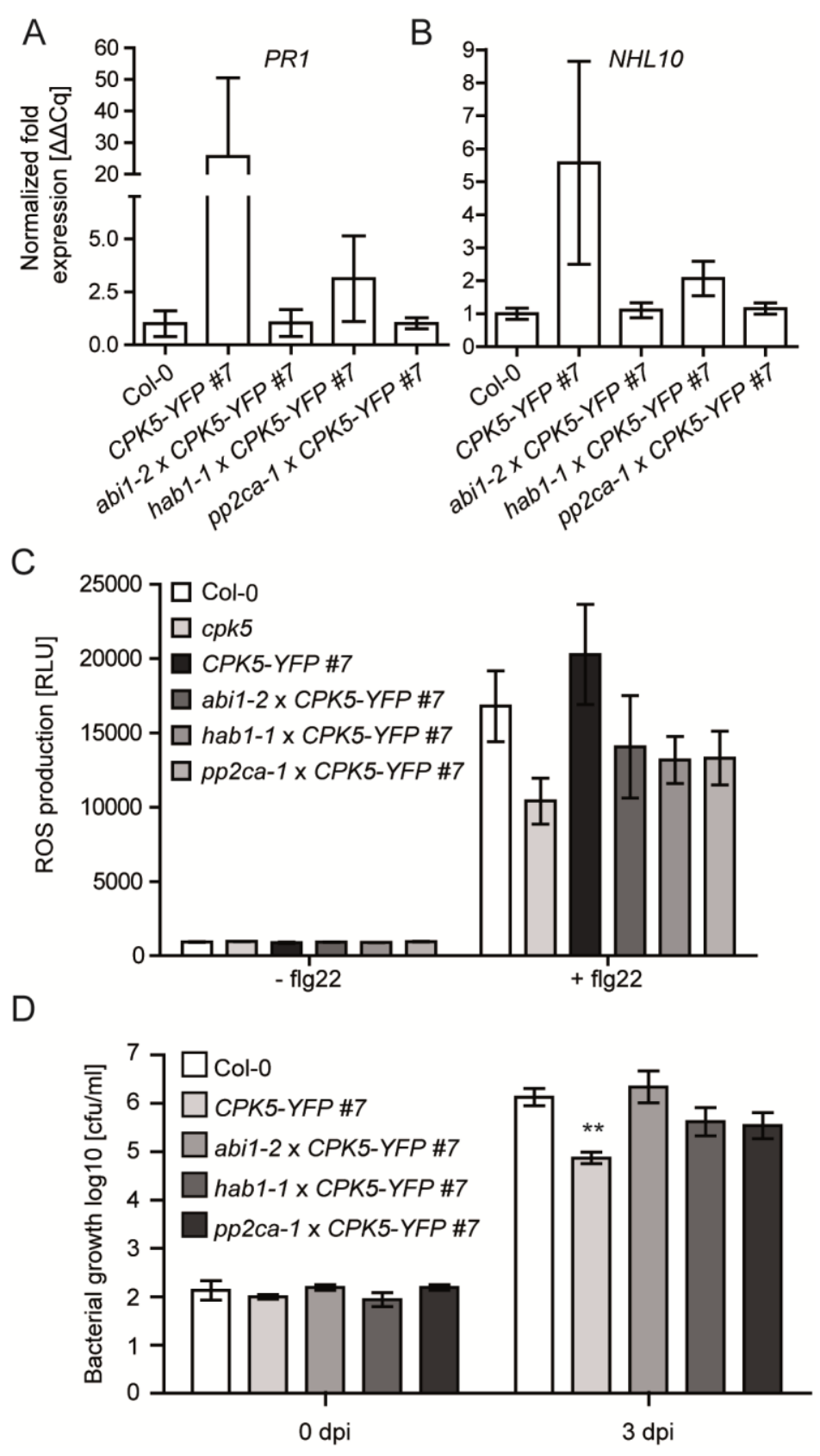

Fig. 2 CPK5-dependent resistance phenotypes are reverted in $p p 2 c$ knock out backgrounds. $(A)+(B)$ Basal transcript levels of defense related marker genes PR1 (A) and NHL1O (B) in Col-0, the overexpression line CPK5-YFP \#7 and crossings of this line to PP2C knockout mutants abil-2, habl-1 and pp2ca-1 $(\mathrm{n}=4-14)$. (C) ROS levels measured with a luminol-based assay in absence or presence of $200 \mathrm{nM}$ flg22 over $1 \mathrm{~h}$ in the lines Col-0, cpk5, CPK5-YFP \#7 and crossing lines as in $(A)(\mathrm{n}=4-12)$. Values represent mean $\pm \mathrm{SD}$ of $\mathrm{n}$ biological replicates. (D) Bacterial growth of Pto DC3000 tested directly and 3 days after infection in overexpression lines as in $(A)$ and $(B)(\mathrm{n}=8-14)$. Data was checked for statistical differences using one-way ANOVA Dunnett post-test. Significant differences $* *=p<0.01$. 

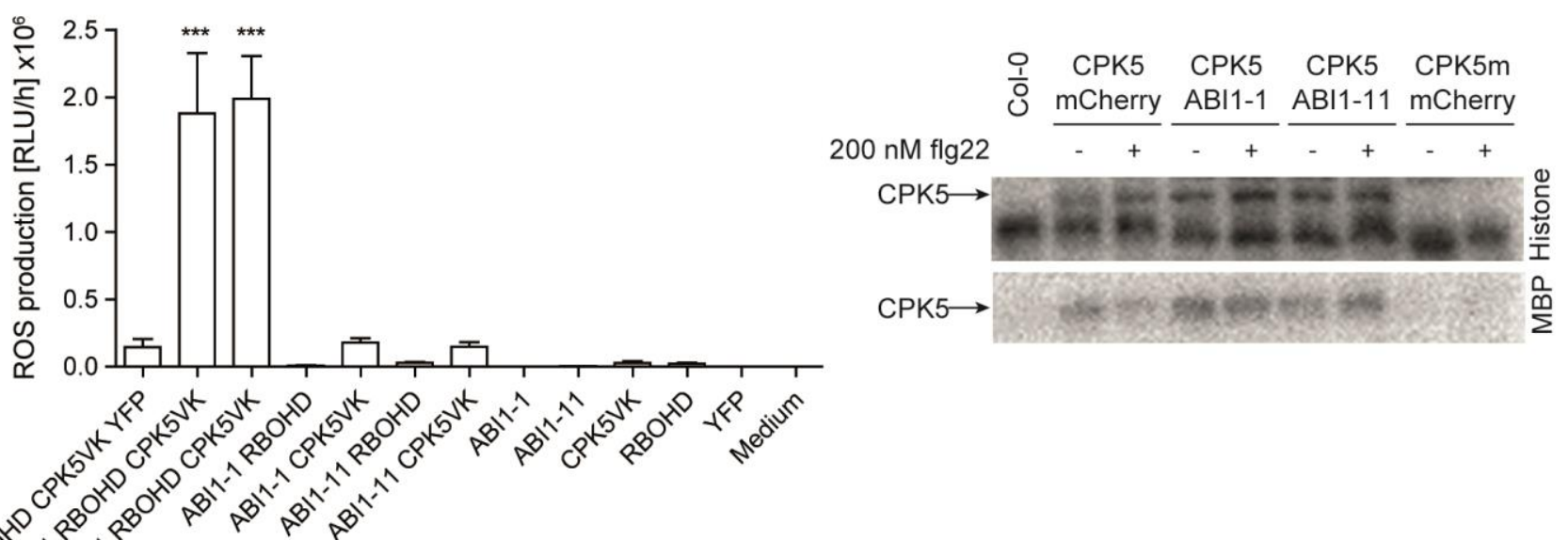

स्तु

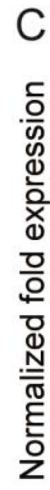
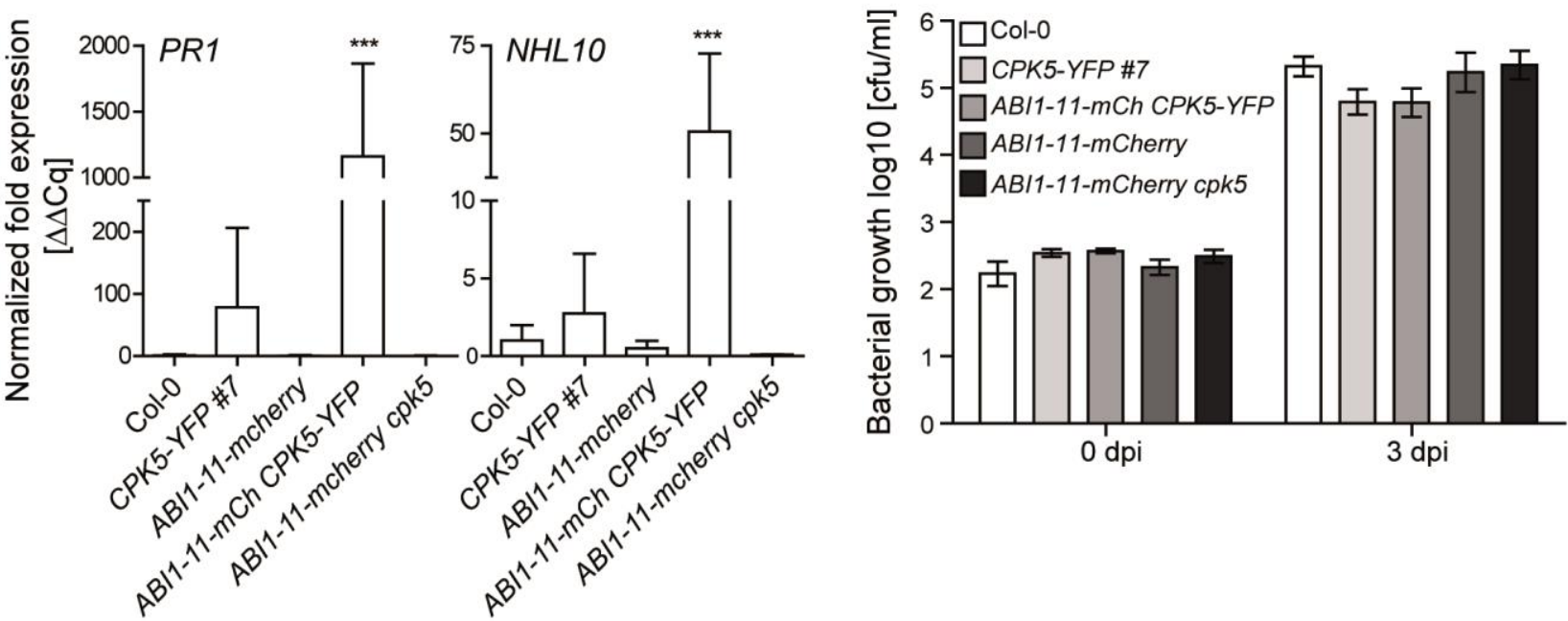

Fig. 3

ABI1 promotes CPK5-dependent immune responses and stabilizes kinase activity. $(A)$ ABI1-1- and ABI1-11-mcherry were transiently co-expressed with RBOHD-YFP and CPK5 VKStrepII in $N$. benthamiana $(\mathrm{n}=8)$. ROS were measured with a luminol-based assay over $1 \mathrm{~h}$. Values represent mean \pm SD of $n$ biological replicates. Data was checked for statistical differences using oneway ANOVA (Bonferroni post-test, significant differences $* * *=p<0.001$ ). (B) In gel kinase assay with protein extracts from Arabidopsis mesophyll protoplast transiently coexpressing CPK5-StrepII with ABI1-1-mcherry, ABI1-11-mcherry or mcherry-tag alone as well as CPK5m-Strep as a negative control. Protoplast were treated with $200 \mathrm{nM}$ flg22 or a control treatment for $10 \mathrm{~min}$. (C) Basal transcript levels of defense related marker genes $P R 1$ and $N H L 10$ in Col-0 and the overexpression lines CPK5-YFP \#7, ABI1-11-mcherry CPK5-YFP, ABI1-11-mcherry and ABI1-11-mcherry cpk5 ( $\mathrm{n}=4) .(D)$ Bacterial growth of Pto DC3000 tested directly and 3 days after infection in overexpression lines as mentioned in $(C)(\mathrm{n}=14)$ Data was checked for statistical differences using one-way ANOVA (Bonferroni post-test). Significant differences $* * *=p<0.001$. 


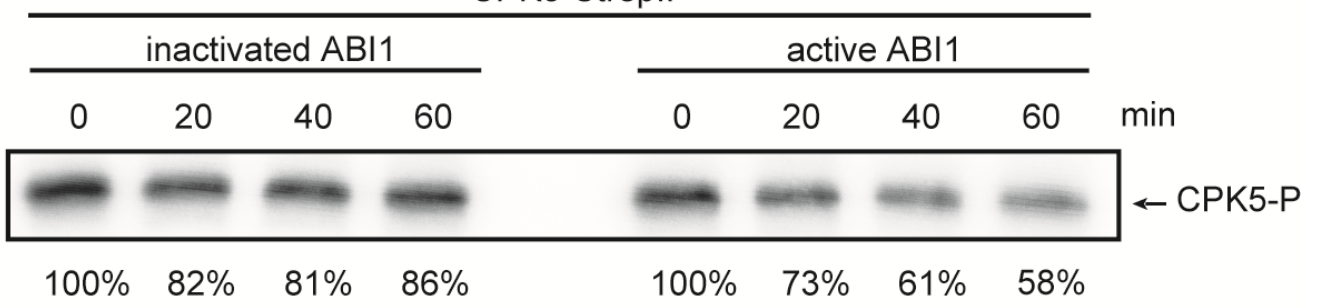

B

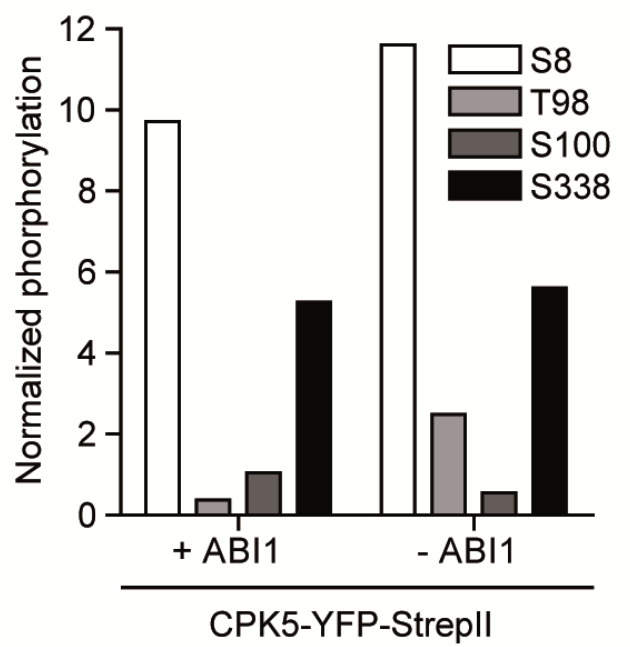

C

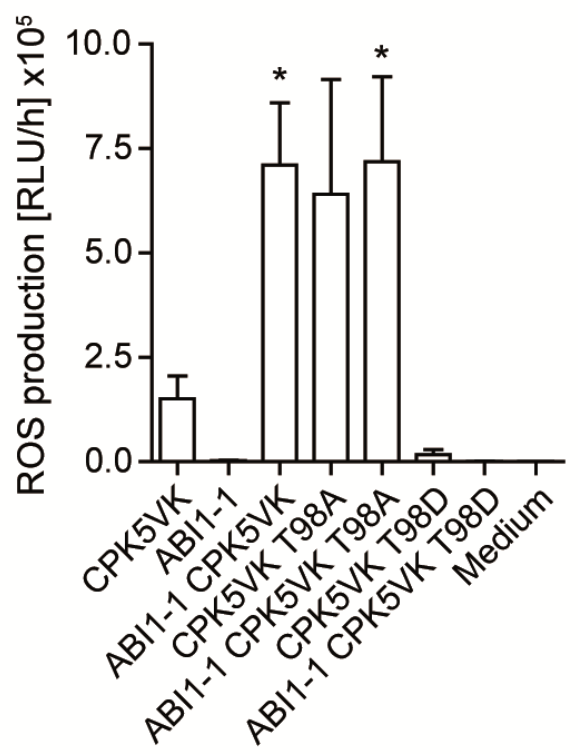

Fig. 4 Regulation of CPK5 activity and function by an ABI1-dependent protein mark: (A) In vitro kinase assay with $200 \mathrm{ng}$ recombinant CPK5-StrepII co-incubated with $100 \mathrm{ng}$ recombinant ABI1-StrepII (active of heat-inactivated). CPK5 was inactivated with K252-a previous to coincubation with ABI1. Percentage corresponds to amount of autophosphorylated kinase after incubation with ABI1 phosphatase. $(B)$ Quantitative analysis of in vitro phosphorylation of CPK5StrepII/CPK5-YFP-StrepII in the absence and presence of ABI1 as in $(A)$. (C) Transient ROS assay with phosphomutant (T98A) and phosphomimic (T98D) versions at position 98 of CPK5 VK. ROS were measured with a luminol-based assay over $1 \mathrm{~h}(\mathrm{n}=8)$. Values represent mean \pm standard error of $\mathrm{n}$ biological replicates. Data was checked for statistical differences using one-way ANOVA (Dunnett post-test). Significant differences against CPK5VK $*=p<0.05$. 
A
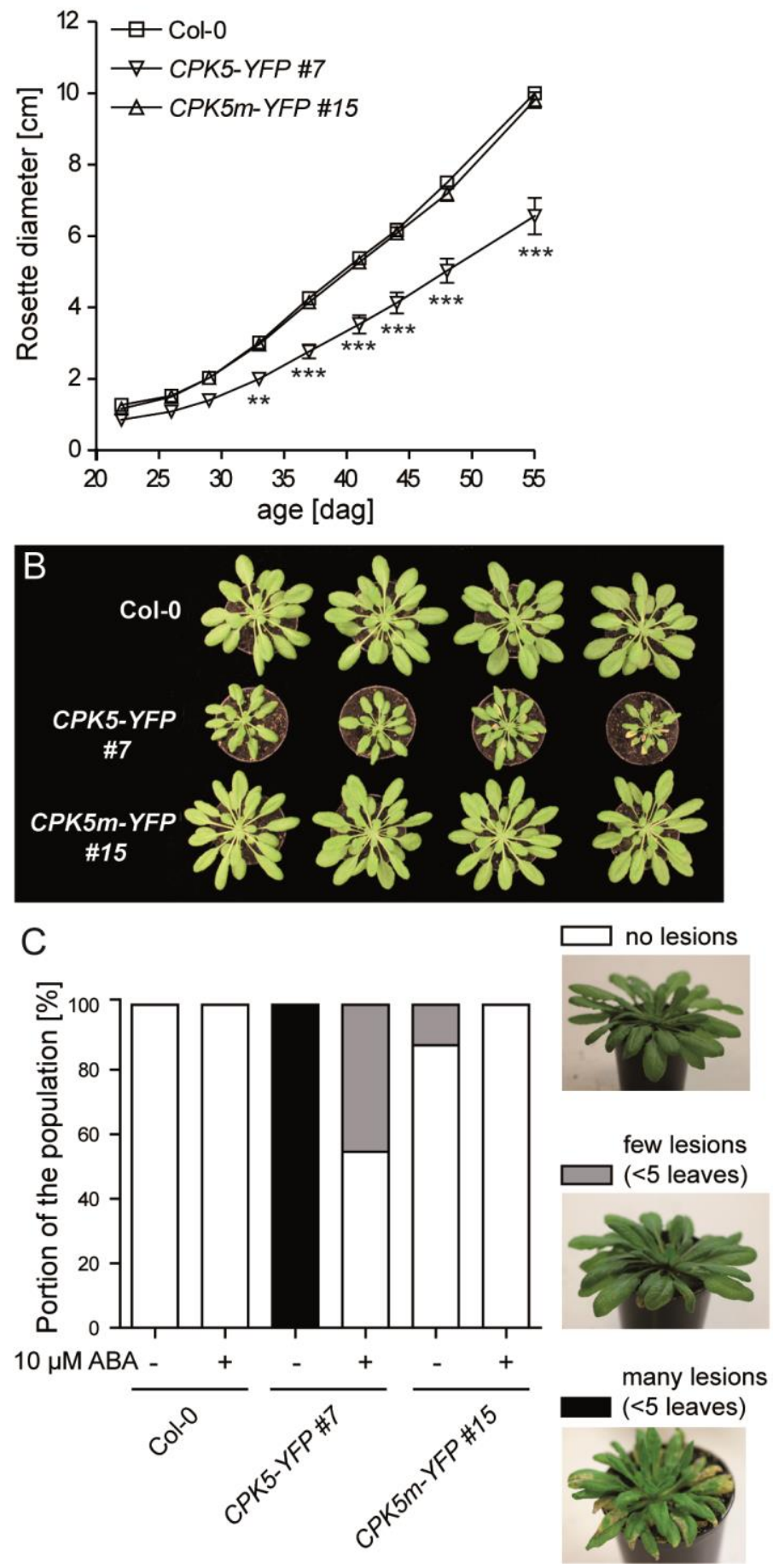

Fig. S1 Growth and lesion mimic phenotype of CPK5-YFP overexpression lines. $(A)$ Rosette diameter $(\mathrm{n}=10-14)$ and $(B)$ phenotype of 8 week old plants of the lines Col-0, CPK5-YFP \#7 und $C P K 5 m-Y F P \# 15$. Values represent mean \pm standard error of $\mathrm{n}$ biological replicates. Data in $(A)$ was checked for statistical differences using two-way ANOVA (Bonferroni post-test, significant differences $* *=\mathrm{p}<0.01$ and $* * *=\mathrm{p}<0.001)$. $(C)$ Evaluation of lesions of 70 days old plants of the lines Col-0, CPK5-YFP \#7 and CPK5m-YFP \#15 as in $(A)+(B)$ with examples of categories for evaluation of lesions. 


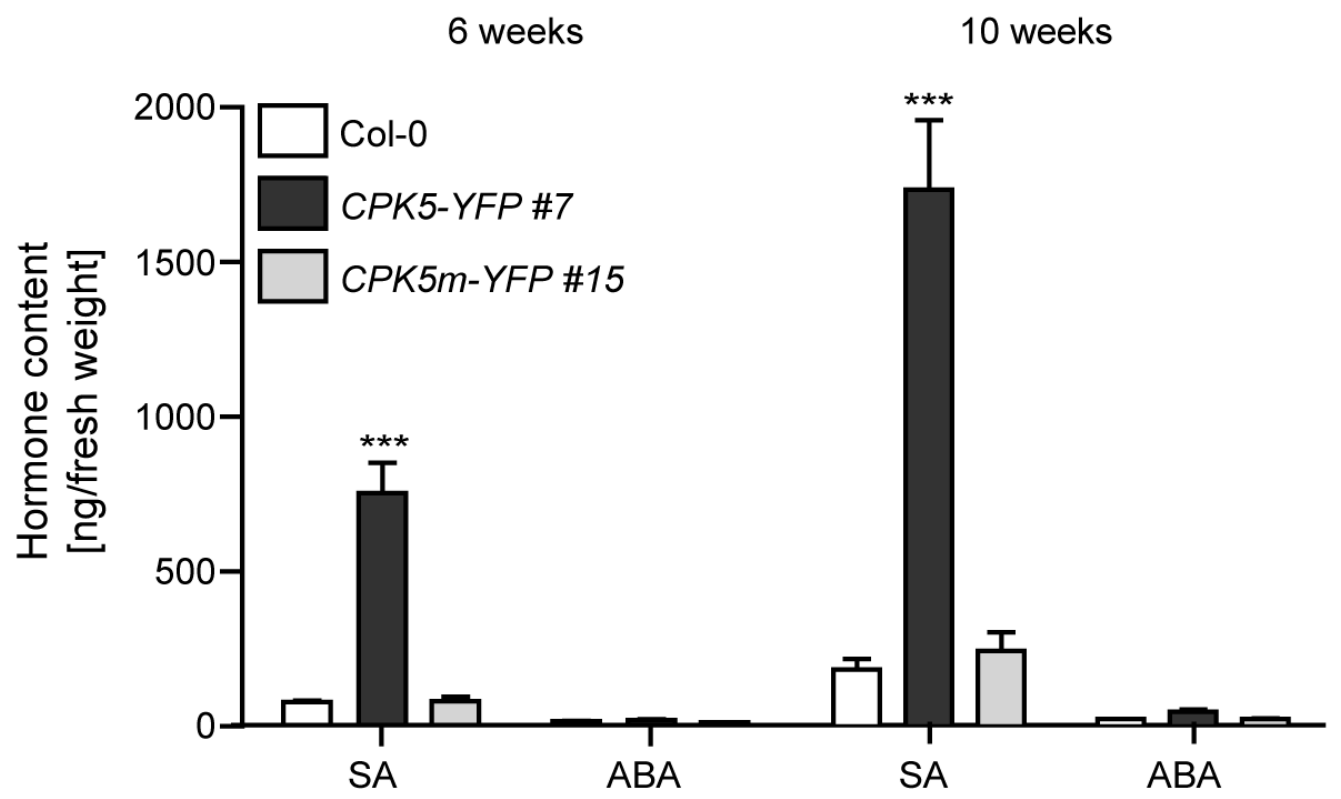

Fig. S2 Basal phytohormone contents in CPK5-YFP lines. Basal SA- and ABA-levels in unstressed 6 and 10 week old plants of the lines Col-0, CPK5-YFP \#7 und CPK5m-YFP \#15 (n = 5-7) measured via UPLC-TOF-MS/MS. Values represent mean \pm standard error of $n$ biological replicates. Data was checked for statistical differences using one-way ANOVA (Bonferroni post-test, significant differences $* * *=p<0.001)$. 


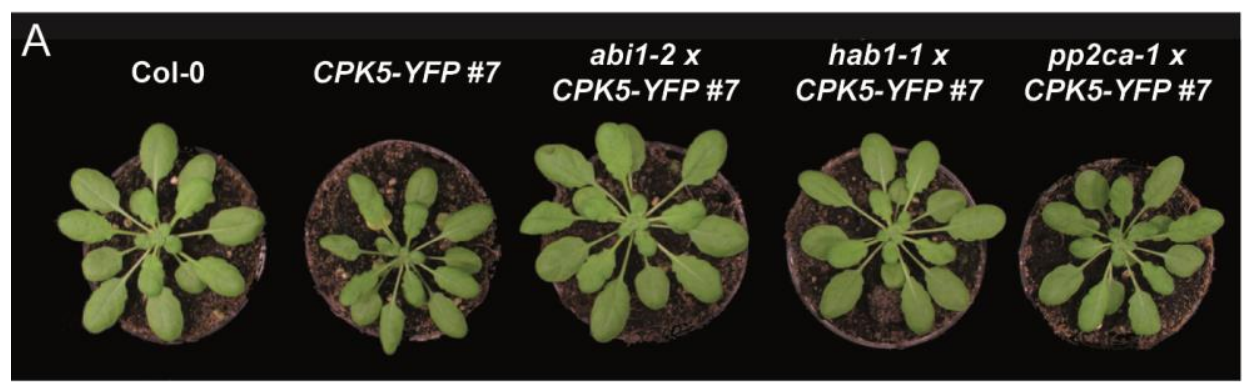

B

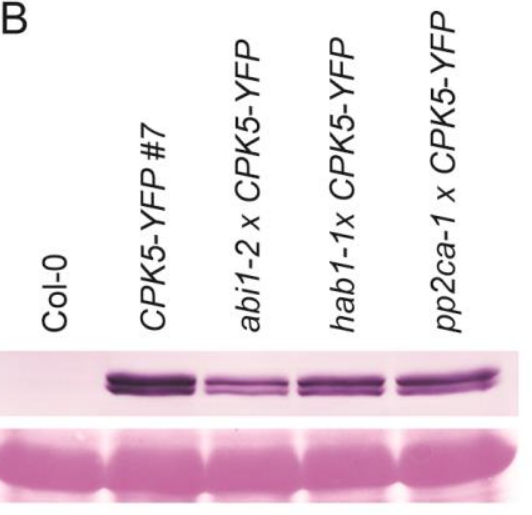

C

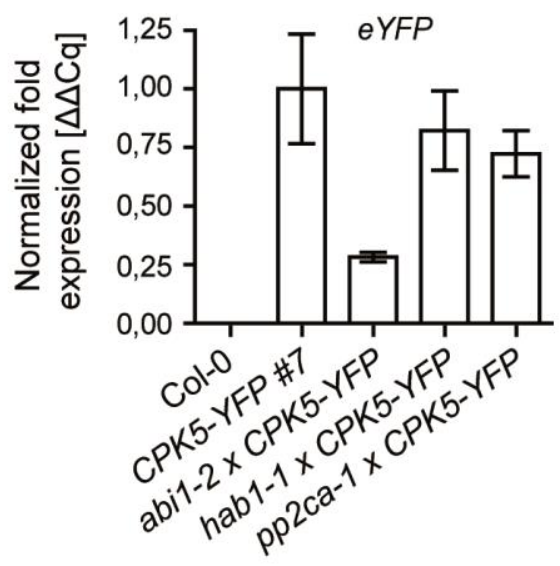

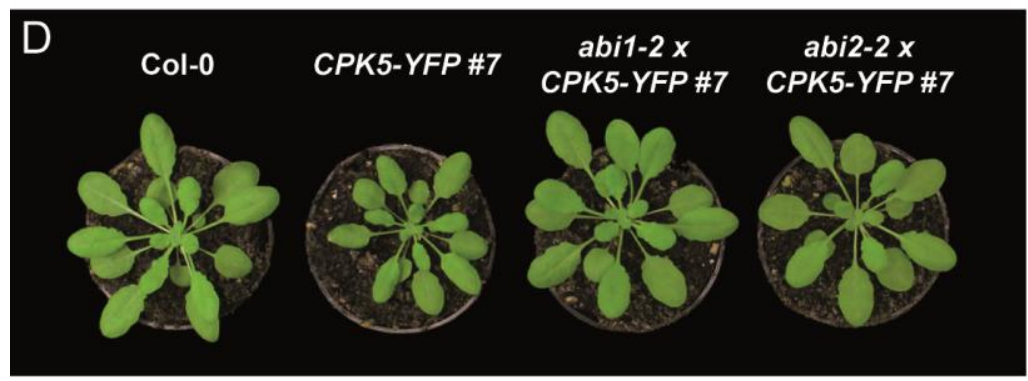

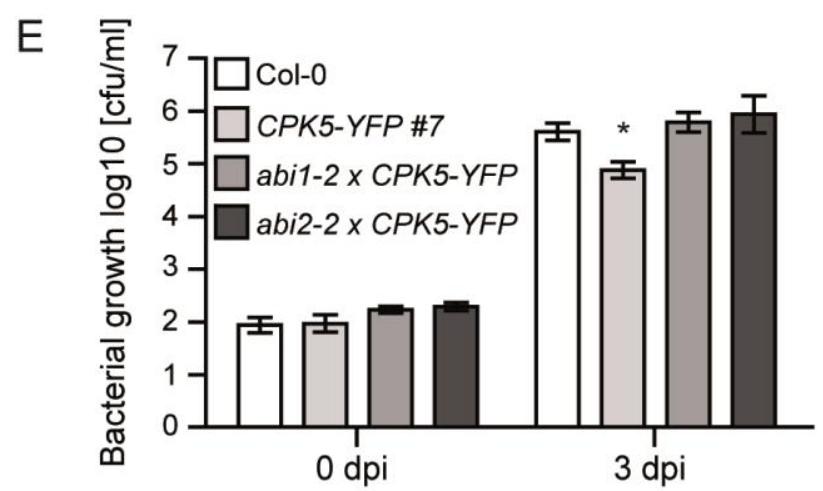

Fig. $\quad$ S3 CPK5-YFP phenotype is reverted in different $p p 2 c$ knock out backgrounds. $(A)$ Phenotype of 6 week old plants of the lines Col-0, CPK5-YFP \#7 and the crossing lines abil-2 x CPK5-YFP \#7, hab1-1 $x$ CPK5-YFP \#7 and pp2ca-1 $x$ CPK5-YFP \#7. (B) Western blot analysis of CPK5-YFP expression in 3 week old plants as in $(A)$ via detection of YFP $(C)$ Transcript levels of YFP in 6 week old plants as mentioned in $(A)(\mathrm{n}=2-4)$. (D) Phenotype of 6 week old plants of the lines Col-0, CPK5-YFP \#7 and the crossing lines abil-2 $x$ CPK5-YFP \#7 and abi2-2 $x$ CPK5-YFP \#7. (E) Bacterial growth of Pto DC3000 tested directly and 3 days after infection in lines as in $(D)(\mathrm{n}=8-16)$. Data was checked for statistical differences using one-way ANOVA (Dunnett post-test). Significant differences $*=p<0.05$. 

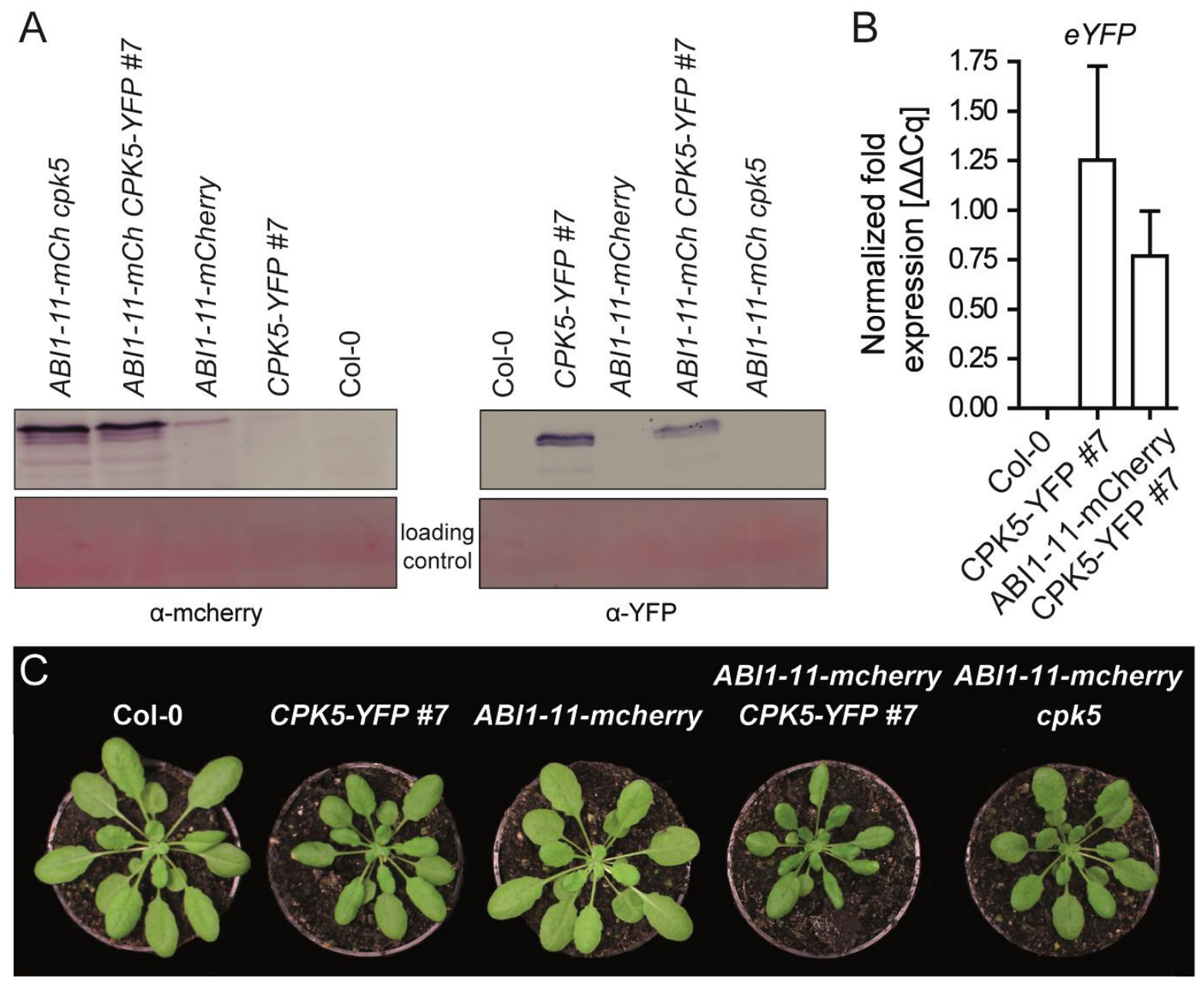

Fig. S4 Phenotype of ABI1-11-mcherry lines. (A) Western blot analysis of protein expression in the lines Col-0, CPK5-YFP \#7, ABI1-11-mcherry, ABI1-11-mcherry CPK5-YFP \#7 and $A B I 1-11$-mcherry cpk5 via detection of mcherry and YFP. $(B)$ Transcript levels of YFP in Col-0, CPK5-YFP \#7 and ABI1-11-mcherry CPK5-YFP \#7 in 6 week old plants $(\mathrm{n}=4)$. (C) Phenotype of 6 week old plants of the lines as mentioned in $(A)$. 


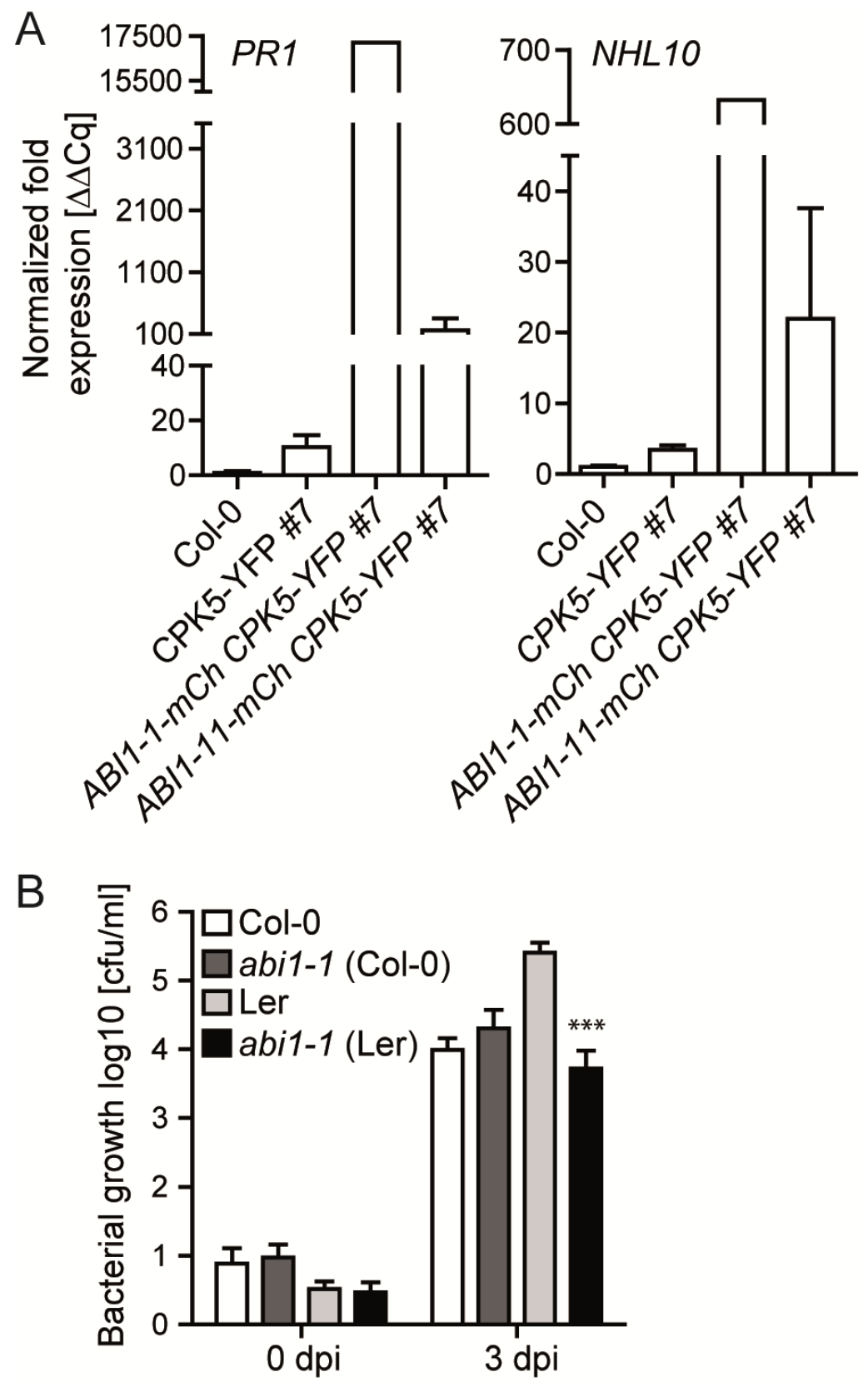

Fig. S5 Characterization of ABI1-1-mcherry and abil-1 expressing plants. (A) Basal transcript levels of PRI and NHL10 in 6 week old plants of the lines Col-0, CPK5-YFP \#7, ABI1-1-mcherry CPK5-YFP \#7 and ABI1-11-mcherry CPK5-YFP \#7 (n = 1-10). Data was checked for statistical differences using one-way ANOVA (Dunnett post-test). (B) Bacterial growth of Pto DC3000 tested directly and 3 days after infection in point mutants of $A B I 1$ in different wild type backgrounds $(n=9-12)$. Data was checked for statistical differences between abil-1 mutant and corresponding wild type using students t-test. Significant differences $* * *=\mathrm{p}<0.001)$. 
A

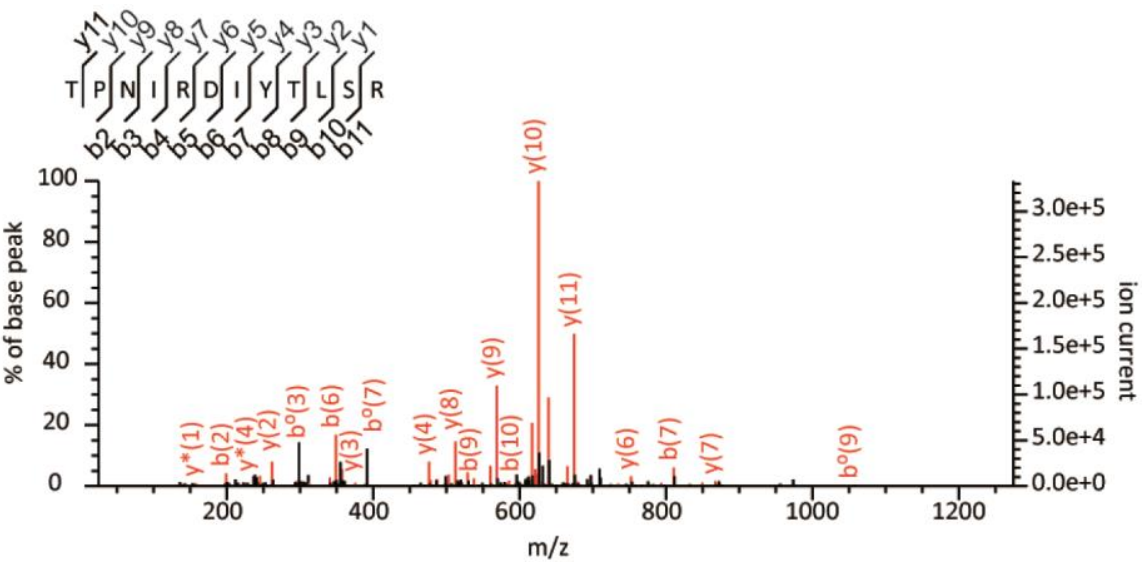

B
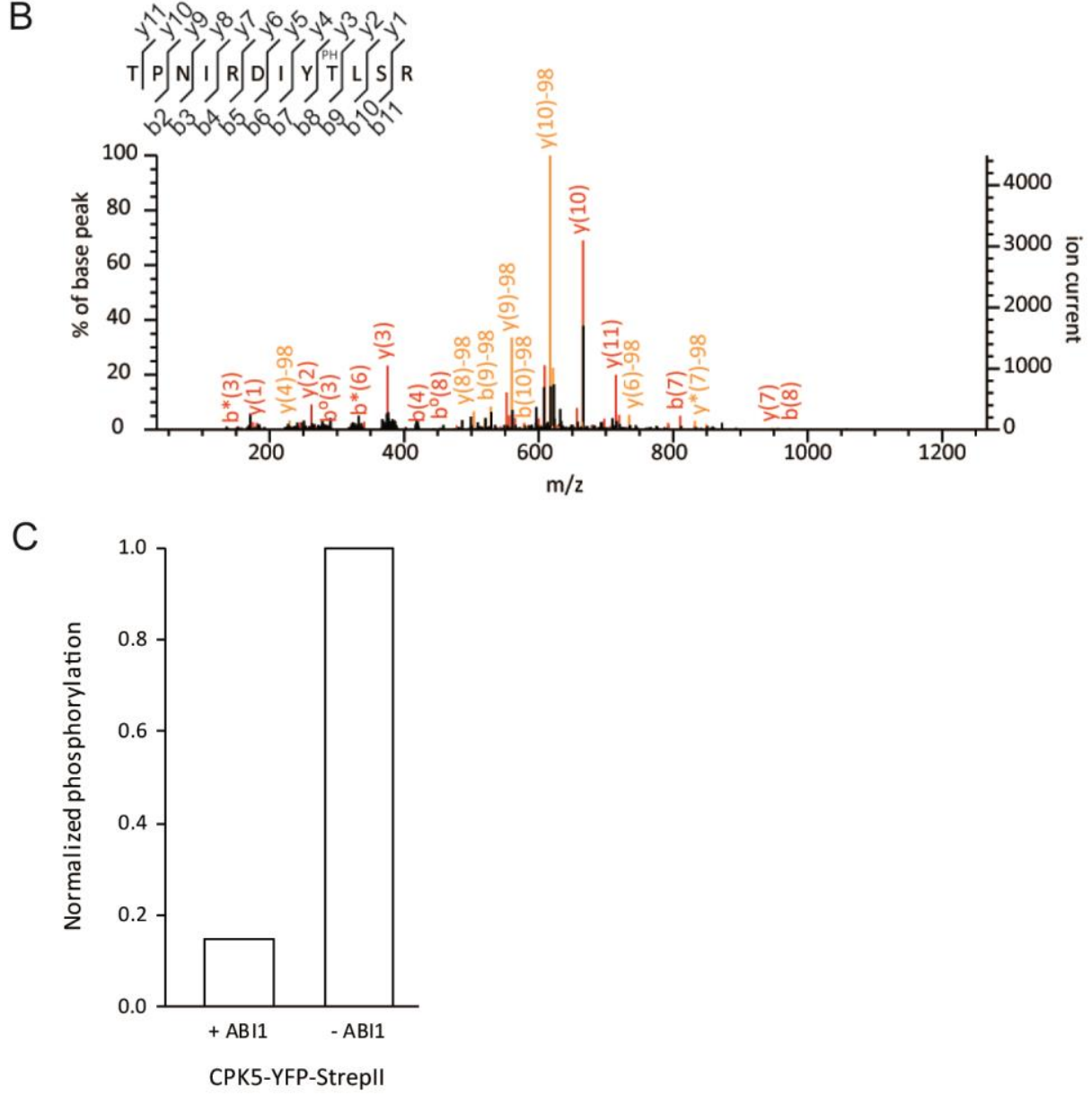

Fig. S6 ABI-dependent phosphorylation sites of CPK5. MS spectra of T98 (A) and T98phos $(B)$. (C) Section of Fig. 4B showing only quantitative analysis of in vitro phosphorylation of $\mathrm{T} 98$ in the absence and presence of $\mathrm{ABI}$. 
Supplementary Information Appendix

Prioritization of abiotic and biotic stress responses by direct linkage of ABI1 phosphatase and CPK5 calcium-dependent protein kinase

Heike Seybold, Jennifer Bortlik, Benjamin Conrads, Wolfgang Hoehenwarter, Tina Romeis Corresponding author: Tina Romeis (Email: tina.romeis@ipb-halle.de)

This PDF file includes:

Supplementary text

Figs. S1 to S6

Table S1

Supplementary Information Appendix

\section{SI Appendix Materials and Methods}

\section{Material and Methods}

\section{Plant material}

Arabidopsis thaliana plants were grown on soil with $8 \mathrm{~h}$ light/16h dark cycle, $20{ }^{\circ} \mathrm{C}$ and $60 \%$ relative humidity. If not stated otherwise, all transgenic plants were generated in Col-0 ecotype background. T-DNA insertion lines abil-2 (SALK_072009), hab1-1 (SALK_02104), pp2ca-1 (SALK_28132), abi2-2 (SALK_15166) as well as abil-1 (Col-0 background, dominant mutant) were kindly provided by J. Kudla (WWU Münster) and abil-1 (Ler background, dominant mutant) and the Ler ecotype were kindly provided by E. Grill (TU München). cpk5 (SAIL_657_C06) was obtained from Nottingham Arabidopsis Stock Center (NASC). Transgenic overexpression lines CPK5-YFP \#7 and CPK5m-YFP \#15 were generated previously (6). Nicotiana benthamiana wildtype plants were grown on soil in a greenhouse with 16h light/8h dark cycle.

\section{Generation of mutant constructs}


Generation of the ABI1 point mutation variants ABI1-1 (G180D) and ABI1-11 (G181S) and CPK5 phosphosite mutant, T98A/D, was performed using PCR mutagenesis (for primers see Table S1). ABI1-1 and ABI1-11 were put into the binary vector pXCSnpt-p35S::mCherry. Overexpression lines were generated using floral dipping (60) in the respective background (Col-0, cpk5, CPK5-YFP \#7)

\section{In-gel kinase assay and Western-blot}

In-gel kinase assays for in vivo kinase activity and immunoblot analysis of proteins were performed as described in detail previously (61).

\section{Measurement of transcript levels by qRT-PCR analysis}

RNA was extracted from ground leaf tissue using the Trizol method. $2 \mu \mathrm{g}$ of total RNA was treated with RNase-free DNase (Fermentas) and mRNA was reverse transcribed with SuperscriptIII SuperMix (Invitrogen) according to the manufacturer's protocols. Real-time quantitative PCR analysis was performed according to the instructions of Power SYBR Green PCR Master Mix (Applied Biosystems) using the CFX96 system (Bio-Rad). Post-amplification dissociation curves were used for evaluation of amplification specificity. Gene expression was quantified using ACTIN2 (At3g18780). Primer sequences are listed in Table S1.

\section{ROS measurement}

Production of reactive oxygen species (ROS) was monitored using a leaf disc assay described previously (6). Summarized, ROS production in A. thaliana was tested as flg22-induced oxidative burst. In $N$. benthamiana ROS was measured in the absence of flg22 3 days after $A$. tumefaciens-mediated transformation with constitutively active CPK5 VK constructs.

\section{ABA treatment}

Plants were treated with ABA solutions by spraying whole rosettes with a manual aerosol until all leaves were moistened. Short-term $(6 \mathrm{~h})$ and intermediate-term (14 days) effects of ABA on CPK5-YFP activity and lesion phenotype were tested after spray treatment of plants with $3 \mu \mathrm{M}$ ABA (in $30 \mu \mathrm{M}$ MES, pH 5.7) every second day. Long-term effects of ABA treatment on the CPK5-YFP lesion phenotype were monitored over a growth period of 10 weeks under continuous treatment with $10 \mu \mathrm{M}$ ABA (in $30 \mu \mathrm{M}$ MES, pH 5.7) every second day.

\section{Bacterial growth}


Growth of $P$. syringae pv. tomato DC3000 (Pto DC3000) was monitored as described before (6). Summarized, 6-week old plants were syringe infiltrated with $10^{4} \mathrm{cfu} / \mathrm{ml}$ Pto DC3000 in 10 $\mathrm{mM} \mathrm{MgCl} 2$. Bacterial growth was tested three days after inoculation by serial dilution plating of leaf disc extracts.

\section{Phytohormone analysis with UPLC-TOF-MS/MS}

Plant extracts were prepared after a modified method described previously (Pan et al. 2008). 1 $\mathrm{ml}$ extraction buffer (100\% ethyl acetate, deuterized internal standard mix), $500 \mathrm{mg}$ Matrix D (Fast-Prep Homogenizer) and 2 steal beads were added to 200-300 mg plant material and mixed at RT for $1 \mathrm{~min}$ at $5 \mathrm{~m} / \mathrm{sec}$ in 24/2 FastPrep ${ }^{\circledR}-24$ (MP Biomedicals) followed by $30 \mathrm{sec}$ incubation on ice. $1 \mathrm{ml}$ supernatant (after centrifugation at $16.000 \mathrm{x} \mathrm{g}, 4{ }^{\circ} \mathrm{C}, 10 \mathrm{~min}$ ) was vacuum-concentrated. The remaining pellet was dissolved in $1 \mathrm{ml}$ extraction buffer, centrifuged twice and the supernatant was combined with the first one and again concentrated for 20-30 min. The pellet was dissolved in $400 \mu 1$ Re-eluation buffer (70\% Methanol, 0.1 formaldehyde) and mixed for $10 \mathrm{~min}$ at RT.

Samples were loaded on a C18 column (Acquinity UPLC BEH-C18, length $5 \mathrm{~cm}$, ID $2.1 \mathrm{~mm}$, particle size $1,7 \mu \mathrm{m}$ ) for hormone separation at $4{ }^{\circ} \mathrm{C}$. Elution was started with constant $30 \%$ eluent B (Methanol with 0.1 formic acid, eluent A was water with $0.1 \%$ formic acid) followed by a linear gradient to $90 \%$ of eluent B for $3.5 \mathrm{~min}$ and constant $90 \%$ eluent B for another 3.5 min. Eluent B was decreased to $30 \%$ for 1 min and kept at $30 \%$ for additional 3 min. Flow rate was $0.25 \mathrm{ml} / \mathrm{min}$ with an injection volume of $7 \mu$ per sample. Synapt ${ }^{\circledR}$ G2-S HDMS ESIMS/ MS (Waters) was used for detection of phytohormones in negative ion mode. Electrospray ionization conditions were capillary tension $2.50 \mathrm{kV}$, vaporizer 6.0 bar, desolvatation of gas flow rate $500 \mathrm{l} / \mathrm{h}$, source temp $80{ }^{\circ} \mathrm{C}$, desolvatation temp $150{ }^{\circ} \mathrm{C}$. The specific fragment sizes were $\mathrm{SA}-\mathrm{m} / \mathrm{z} 93, \mathrm{ABA}-\mathrm{m} / \mathrm{z} 153$.

\section{Phosphoproteomics}

Amino acid residue specific phosphorylation of AtCPK5 was mapped by liquid chromatography on-line with high resolution accurate mass MS (HR/AM LC-MS). Proteins separated by SDS-PAGE were subjected to in-gel tryptic digestion. The resulting peptides were separated using C18 reverse phase chemistry employing a pre-column (EASY column SC001, length $2 \mathrm{~cm}$, ID $100 \mu \mathrm{m}$, particle size $5 \mu \mathrm{m}$ ) in line with an EASY column SC200 with a length of $10 \mathrm{~cm}$, an inner diameter (ID) of $75 \mu \mathrm{m}$ and a particle size of $3 \mu \mathrm{mon}$ an EASY-nLC II (all from Thermo Fisher Scientific). Peptides were eluted into a Nanospray Flex ion source (Thermo 
Fisher Scientific) with a 90 min gradient increasing from $5 \%$ to $40 \%$ acetonitrile in $\mathrm{ddH}_{2} \mathrm{O}$ with a flow rate of $300 \mathrm{nl} / \mathrm{min}$ and electrosprayed into an OrbitrapVelos Pro mass spectrometer (Thermo Fisher Scientific). The source voltage was set to $1.9 \mathrm{kV}$, the S Lens RF level to $50 \%$. The delta multipole offset was -7.00. The AGC target value was set to $1 \mathrm{e} 06$ and the maximum injection time ( $\max$ IT) to $500 \mathrm{~ms}$ in the Orbitrap. The parameters were set to $1 \mathrm{e} 04$ and $100 \mathrm{~ms}$ in the LTQ with an isolation width of 2 Da for precursor isolation and MS/MS scanning. Peptides were analyzed by a targeted data acquisition (TDA) scan strategy with inclusion list to specifically select and isolate AtCPK5 phosphorylated peptides for MS/MS peptide sequencing. Multi stage activation (MSA) was applied to further fragment ion peaks resulting from neutral loss of the phosphate moiety by dissociation of the high energy phosphate bond to generate $b$ - and $y$ - fragment ion series rich in peptide sequence information.

MS/MS spectra were used to search the TAIR10 database (ftp://ftp.arabidopsis.org, 35394 sequences, 14486974 residues) with the Mascot software v.2.5 linked to Proteome Discoverer v.1.4. The enzyme specificity was set to trypsin and two missed cleavages were tolerated. Carbamidomethylation of cysteine was set as a fixed modification and oxidation of methionine and phosphorylation of serine, threonine and tyrosine as variable modifications. The precursor tolerance was set to $7 \mathrm{ppm}$ and the product ion mass tolerance was set to $0.8 \mathrm{Da}$. A decoy database search was performed to determine the peptide spectral match (PSM) and peptide identification false discovery rates (FDR). Peptides identifications with an ion score of 24 surpassing the Mascot significance threshold $(\mathrm{p}<0.05)$ which corresponded to a peptide identification FDR of $<4.55 \%$ and a PSM FDR of $1.14 \%$, were accepted. A transferred FDR specifically for phosphopeptide PSMs was calculated according to [Fu et al Molecular and Cellular Proteomics 2014 13: 1359-1368 DOI 10.1074/mcp.O113.030189] and was $4.47 \%$ ThephosphoRS module was used to specifically map phosphorylation to amino acid residues within the primary structure of phosphopeptides.

Table S1 Primer for PCR-Mutagenesis

\begin{tabular}{ll}
\hline abi1-1 G180D fw & GGTGTTTACGACGGCCATGACGGTTCTCAGGTAGCG \\
\hline abi1-1 G180D rev & CGCTACCTGAGAACCGTCATGGCCGTCGTAAACACC \\
abi1-11 G181S fw & GACGGCCATGGCAGTTCTCAGGTAGCG \\
abi1-11 G181S rev & CGCTACCTGAGAACTGCCATGGCCGTC \\
CPK5 T98A fw & CCAAACATTCGTGATATCTATGCCCTTAGCCGC
\end{tabular}




\section{CPK5 T98A rev CCTAGCTTGCGGCTAAGGGCATAGATATCACG \\ CPK5 T98D fw CCAAACATTCGTGATATCTATGACCTTAGCCGC \\ CPK5 T98D rev CCTAGCTTGCGGCTAAgGTCATAGATATCACG}

\section{Primer für qRT-PCR}

\begin{tabular}{|c|c|c|}
\hline Actin fw & At3g 18780 & TCCCTCAGCACATTCCAGCAGAT \\
\hline Actin rev & & AACGATTCCTGGACCTGCCTCATC \\
\hline ICS1 fw & At1g74710 & TTCTCAATTGGCAGGGAGAC \\
\hline ICS1 rev & & AAGCCTTGCTTCTTCTGCTG \\
\hline NHL10 fw & At2g35980 & TTCCTGTCCGTAACCCAAAC \\
\hline NHL10 rev & & CCCTCGTAGTAGGCATGAGC \\
\hline PR1 fw & At2g14610 & CATGGGACCTACGCCTACC \\
\hline PR1 rev & & TTCTTCCCTCGAAAGCTCAA \\
\hline YFP fw & & CCGCCATGCCCGAAGGCTAC \\
\hline YFP rev & & ACCTCGGCGCGGGTCTTGTA \\
\hline
\end{tabular}

\section{References}

1. Seybold $\mathrm{H}$ et al. (2014) $\mathrm{Ca}^{2+}$ signalling in plant immune response:. From pattern recognition receptors to Ca2+ decoding mechanisms. The New phytologist 204:782-790.

2. Hilker M et al. (2016) Priming and memory of stress responses in organisms lacking a nervous system. Biological reviews of the Cambridge Philosophical Society 91:1118-1133.

3. Hake K, Romeis T (2019) Protein kinase-mediated signalling in priming:. Immune signal initiation, propagation, and establishment of long-term pathogen resistance in plants. Plant, cell \& environment 42:904-917.

4. Bürger M, Chory J (2019) Stressed Out About Hormones:. How Plants Orchestrate Immunity. Cell host \& microbe 26:163-172.

5. Boudsocq $\mathrm{M}$ et al. (2010) Differential innate immune signalling via $\mathrm{Ca}^{2+}$ sensor protein kinases. Nature 464:418-422.

6. Dubiella U et al. (2013) Calcium-dependent protein kinase/NADPH oxidase activation circuit is required for rapid defense signal propagation. Proceedings of the National Academy of Sciences of the United States of America 110:8744-8749.

7. Kadota Y et al. (2014) Direct regulation of the NADPH oxidase RBOHD by the PRR-associated kinase BIK1 during plant immunity. Molecular cell 54:43-55. 
8. Li L et al. (2014) The FLS2-associated kinase BIK1 directly phosphorylates the NADPH oxidase RbohD to control plant immunity. Cell host \& microbe 15:329-338.

9. Guerra T et al. (2019) Calcium-dependent protein kinase 5 links calcium signaling with Nhydroxy-1-pipecolic acid- and SARD1-dependent immune memory in systemic acquired resistance. The New phytologist.

10. Liu N et al. (2017) CALCIUM-DEPENDENT PROTEIN KINASE5 Associates with the Truncated NLR Protein TIR-NBS2 to Contribute to exo70B1-Mediated Immunity. The Plant cell 29:746-759.

11. Harmon AC, Gribskov M, Harper JF (2000) CDPKs - a kinase for every $\mathrm{Ca}^{2+}$ signal? Trends in plant science 5:154-159.

12. Harper JF, Breton G, Harmon A (2004) Decoding $\mathrm{Ca}^{2+}$ signals through plant protein kinases. Annual review of plant biology 55:263-288.

13. Boudsocq M, Sheen J (2013) CDPKs in immune and stress signaling. Trends in plant science 18:30-40.

14. Liese A, Romeis T (2013) Biochemical regulation of in vivo function of plant calcium-dependent protein kinases (CDPK). Biochimica et biophysica acta 1833:1582-1589.

15. Yip Delormel T, Boudsocq M (2019) Properties and functions of calcium-dependent protein kinases and their relatives in Arabidopsis thaliana. The New phytologist 224:585-604.

16. Harper JF, Huang JF, Lloyd SJ (1994) Genetic identification of an autoinhibitor in CDPK, a protein kinase with a calmodulin-like domain. Biochemistry 33:7267-7277.

17. Yoo BC, Harmon AC (1996) Intramolecular binding contributes to the activation of CDPK, a protein kinase with a calmodulin-like domain. Biochemistry 35:12029-12037.

18. Romeis T, Ludwig AA, Martin R, Jones JD (2001) Calcium-dependent protein kinases play an essential role in a plant defence response. The EMBO journal 20:5556-5567.

19. Ludwig AA et al. (2005) Ethylene-mediated cross-talk between calcium-dependent protein kinase and MAPK signaling controls stress responses in plants. Proceedings of the National Academy of Sciences of the United States of America 102:10736-10741.

20. Witte C-P et al. (2010) Tobacco calcium-dependent protein kinases are differentially phosphorylated in vivo as part of a kinase cascade that regulates stress response. The Journal of biological chemistry 285:9740-9748.

21. Boudsocq M, Droillard M-J, Regad L, Laurière C (2012) Characterization of Arabidopsis calciumdependent protein kinases. Activated or not by calcium? The Biochemical journal 447:291-299.

22. Hubbard KE, Nishimura N, Hitomi K, Getzoff ED, Schroeder JI (2010) Early abscisic acid signal transduction mechanisms:. Newly discovered components and newly emerging questions. Genes \& development 24:1695-1708.

23. Lee SC, Luan S (2012) ABA signal transduction at the crossroad of biotic and abiotic stress responses. Plant, cell \& environment 35:53-60.

24. Fujii $\mathrm{H}$ et al. (2009) In vitro reconstitution of an abscisic acid signalling pathway. Nature 462:660-664.

25. Edel KH, Kudla J (2016) Integration of calcium and ABA signaling. Current opinion in plant biology 33:83-91. 
26. Park S-Y et al. (2009) Abscisic acid inhibits type $2 \mathrm{C}$ protein phosphatases via the PYR/PYL family of START proteins. Science (New York, N.Y.) 324:1068-1071.

27. Ma Y et al. (2009) Regulators of PP2C phosphatase activity function as abscisic acid sensors. Science (New York, N.Y.) 324:1064-1068.

28. Soon F-F et al. (2012) Molecular mimicry regulates ABA signaling by SnRK2 kinases and PP2C phosphatases. Science (New York, N.Y.) 335:85-88.

29. Geiger D et al. (2009) Activity of guard cell anion channel SLAC1 is controlled by drought-stress signaling kinase-phosphatase pair. Proceedings of the National Academy of Sciences of the United States of America 106:21425-21430.

30. Geiger D et al. (2010) Guard cell anion channel SLAC1 is regulated by CDPK protein kinases with distinct $\mathrm{Ca}^{2+}$ affinities. Proceedings of the National Academy of Sciences of the United States of America 107:8023-8028.

31. Assmann SM, Jegla T (2016) Guard cell sensory systems. Recent insights on stomatal responses to light, abscisic acid, and $\mathrm{CO}_{2}$. Current opinion in plant biology 33:157-167.

32. Henfling, J. W. D. M. (1980) Effect of Abscisic Acid on Rishitin and Lubimin Accumulation and Resistance to Phytophthora infestans and Cladosporium cucumerinum in Potato Tuber Tissue Slices. Phytopathology 70:1074.

33. Ward EW, Cahill DM, Bhattacharyya MK (1989) Abscisic Acid Suppression of Phenylalanine Ammonia-Lyase Activity and mRNA, and Resistance of Soybeans to Phytophthora megasperma f.sp. glycinea. Plant physiology 91:23-27.

34. McDonald KL, Cahill DM (1999) Influence of Abscisic Acid and the Abscisic Acid Biosynthesis Inhibitor, Norflurazon, on Interactions Between Phytophthora sojae and Soybean (Glycine max). European Journal of Plant Pathology 105:651-658.

35. Mohr PG, Cahill DM (2003) Abscisic acid influences the susceptibility of Arabidopsis thaliana to Pseudomonas syringae pv. tomato and Peronospora parasitica. Functional Plant Biol. 30:461.

36. Thaler JS, Bostock RM (2004) Interactions between Abscisic-acid-mediated responses and plant resistance to pathogens and insects. Ecology 85:48-58.

37. Torres-Zabala M de et al. (2007) Pseudomonas syringae pv. tomato hijacks the Arabidopsis abscisic acid signalling pathway to cause disease. The EMBO journal 26:1434-1443.

38. Mohr PG, Cahill DM (2007) Suppression by ABA of salicylic acid and lignin accumulation and the expression of multiple genes, in Arabidopsis infected with Pseudomonas syringae pv. tomato. Functional \& integrative genomics 7:181-191.

39. Torres Zabala M de, Bennett MH, Truman WH, Grant MR (2009) Antagonism between salicylic and abscisic acid reflects early host-pathogen conflict and moulds plant defence responses. The Plant journal : for cell and molecular biology 59:375-386.

40. Peng Z et al. (2019) Xanthomonas translucens commandeers the host rate-limiting step in ABA biosynthesis for disease susceptibility. Proceedings of the National Academy of Sciences of the United States of America 116:20938-20946.

41. Zeng W, He SY (2010) A prominent role of the flagellin receptor FLAGELLIN-SENSING2 in mediating stomatal response to Pseudomonas syringae pv tomato DC3000 in Arabidopsis. Plant physiology 153:1188-1198. 
42. McLachlan DH, Kopischke M, Robatzek S (2014) Gate control:. Guard cell regulation by microbial stress. The New phytologist 203:1049-1063.

43. Melotto M, Zhang L, Oblessuc PR, He SY (2017) Stomatal Defense a Decade Later. Plant physiology 174:561-571.

44. Liu Y et al. (2019) Anion channel SLAH3 is a regulatory target of chitin receptor-associated kinase PBL27 in microbial stomatal closure. eLife 8.

45. Melotto M, Underwood W, Koczan J, Nomura K, He SY (2006) Plant stomata function in innate immunity against bacterial invasion. Cell 126:969-980.

46. Lim CW, Luan S, Lee SC (2014) A prominent role for RCAR3-mediated ABA signaling in response to Pseudomonas syringae pv. tomato DC3000 infection in Arabidopsis. Plant \& cell physiology 55:1691-1703.

47. Mori IC et al. (2006) CDPKs CPK6 and CPK3 function in ABA regulation of guard cell S-type anion- and $\mathrm{Ca}^{2+}$-permeable channels and stomatal closure. PLoS biology 4:e327.

48. Prodhan MY, Munemasa S, Nahar MN-E-N, Nakamura Y, Murata Y (2018) Guard Cell Salicylic Acid Signaling Is Integrated into Abscisic Acid Signaling via the $\mathrm{Ca}^{2+} / \mathrm{CPK}-D e p e n d e n t$ Pathway. Plant physiology 178:441-450.

49. Berens ML et al. (2019) Balancing trade-offs between biotic and abiotic stress responses through leaf age-dependent variation in stress hormone cross-talk. Proceedings of the National Academy of Sciences of the United States of America 116:2364-2373.

50. Seo DH, Ahn MY, Park KY, Kim EY, Kim WT (2016) The N-Terminal UND Motif of the Arabidopsis U-Box E3 Ligase PUB18 Is Critical for the Negative Regulation of ABA-Mediated Stomatal Movement and Determines Its Ubiquitination Specificity for Exocyst Subunit Exo70B1. The Plant cell 28:2952-2973.

51. Mine A et al. (2017) Pathogen exploitation of an abscisic acid- and jasmonate-inducible MAPK phosphatase and its interception by Arabidopsis immunity. Proceedings of the National Academy of Sciences of the United States of America 114:7456-7461.

52. Yasuda M et al. (2008) Antagonistic interaction between systemic acquired resistance and the abscisic acid-mediated abiotic stress response in Arabidopsis. The Plant cell 20:1678-1692.

53. Ding Y, Dommel M, Mou Z (2016) Abscisic acid promotes proteasome-mediated degradation of the transcription coactivator NPR1 in Arabidopsis thaliana. The Plant journal : for cell and molecular biology 86:20-34.

54. Leung J et al. (1994) Arabidopsis ABA response gene ABI1:. Features of a calcium-modulated protein phosphatase. Science (New York, N.Y.) 264:1448-1452.

55. Meyer K, Leube MP, Grill E (1994) A protein phosphatase 2C involved in ABA signal transduction in Arabidopsis thaliana. Science (New York, N.Y.) 264:1452-1455.

56. Hua D et al. (2012) A plasma membrane receptor kinase, GHR1, mediates abscisic acid- and hydrogen peroxide-regulated stomatal movement in Arabidopsis. The Plant cell 24:2546-2561.

57. Schulz P, Herde M, Romeis T (2013) Calcium-dependent protein kinases:. Hubs in plant stress signaling and development. Plant physiology 163:523-530.

58. Kline KG, Barrett-Wilt GA, Sussman MR (2010) In planta changes in protein phosphorylation induced by the plant hormone abscisic acid. Proceedings of the National Academy of Sciences of the United States of America 107:15986-15991. 
59. Brandt B et al. (2015) Calcium specificity signaling mechanisms in abscisic acid signal transduction in Arabidopsis guard cells. eLife 4.

60. Clough SJ, Bent AF (1998) Floral dip:. A simplified method for Agrobacterium-mediated transformation of Arabidopsis thaliana. The Plant journal : for cell and molecular biology 16:735-743.

61. Seybold H, Boudsocq M, Romeis T (2017) CDPK Activation in PRR Signaling. Methods in molecular biology (Clifton, N.J.) 1578:173-183. 